\title{
A comprehensive investigation of abrasive barrel finishing on hardness and manufacturability of laser-based powder bed fusion hollow components
}

\author{
Mahyar Khorasani $^{1,2} \cdot$ Amir Hossein Ghasemi $^{3} \cdot$ Ehsan Farabi $^{4} \cdot$ Martin Leary $^{1} \cdot$ Ian Gibson $^{2,5} \cdot$ Bernard Rolfe $^{2}$
}

Received: 31 October 2021 / Accepted: 10 February 2022 / Published online: 4 March 2022

(c) The Author(s) 2022

\begin{abstract}
One of the main issues of laser-based powder bed fusion (LB-PBF) parts is surface quality and dimensional deviations, which require post-processing. Conventional post-processing such as turning and milling cannot machine internal surfaces and therefore is not suitable for hollow components. In this paper, Ti-6Al-4 V components with different hollow shapes were printed by LB-PBF and post-processed by centrifugal barrel finishing (CBF). Samples were printed based on Taguchi L18 design of experiments (DoE) on the (L18: $\left.2^{1} \times 3^{3}\right)$ matrix and polished in abrasive solution by porcelain triangular media $2 \times 2 \mathrm{~mm}$. The effect of process parameters including rotation direction, speed, time and volumetric percentage of abrasive on hardness and manufacturability, including surface quality, material removal rate (MRR) and dimensional deviation, are discussed. The novelty of this work is the application of this process to clean both the internal and external surfaces of LBPBF parts, where previously it has only been investigated for external surfaces. This paper scrutinized the performance of the CBF on internal geometries, and it was shown for the size of the investigated components, the hexagonal hollow achieved the highest maximum removal rate over the square and circular hollows. In addition, the effect of CBF on plastic deformation and microstructural characterization has been investigated to find the effect of this process on work hardening. The results of this study also show that the rotational speed and the volumetric percentage of the abrasive directly drive the MRR. A higher rotational speed increases the slope of the sliding path and the sliding speed between printed parts and abrasive media, which causes higher cutting and grinding, MRR and media wear rate.
\end{abstract}

Keywords Additive manufacturing · Powder bed fusion - Water abrasive barrel finishing · Process parameters · Manufacturability

\begin{tabular}{llll}
\multicolumn{2}{l}{ Abbreviations } & $R_{a}$ & Average roughness \\
ACBF & Abrasive centrifugal barrel finishing & MoM & Mean of means \\
LB-PBF & Laser-based powder bed fusion & BJT & Binder jetting \\
AM & Additive manufacturing & $R_{m}$ & Media radius \\
MRR & Material removal rate & CBF & Centrifugal barrel finishing
\end{tabular}

Mahyar Khorasani

mahyar.khorasani@rmit.edu.au

Amir Hossein Ghasemi

amir_hosein_ghasemi2012@yahoo.com

Ehsan Farabi

ehsan.farabi@deakin.edu.au

Martin Leary

martin.leary@rmit.edu.au

Ian Gibson

i.gibson@utwente.nl

Bernard Rolfe

bernard.rolfe@deakin.edu.au
1 School of Engineering, Royal Melbourne Institute of Technology, Melbourne, Australia

2 School of Engineering, Deakin University, Waurn Ponds, Victoria, Australia

3 Australian Institute of Science \& Technology, Ashfield, NSW, Australia

4 Institute for Frontier Materials, Deakin University, Waurn Ponds, Victoria, Australia

5 Fraunhofer Project Centre for Complex System Engineering, University of Twente, Enschede, The Netherlands 


$\begin{array}{ll}\text { MANOVA } & \text { Multivariate analysis of variance } \\ \text { DoE } & \text { Design of experiment } \\ \text { OEM } & \text { Original equipment manufacturer } \\ \text { DED } & \text { Directed energy deposition } \\ \text { SEM } & \text { Scanning electron microscope } \\ \text { EBSD } & \text { Electron backscattered diffraction } \\ \text { SNR } & \text { Signal to noise ratio }\end{array}$

\section{Introduction}

The commercial application of additive manufacturing (AM) is rapidly expanding due to freedom in design, the ability to produce complex geometry in a single production process and reduction in labour costs [1-3]. Among all seven AM processes that have been defined by Gibson et al. [4], directed energy deposition (DED), powder bed fusion $(\mathrm{PBF})$ and binder jetting (BJT) are mainly used to process metal components. LB-PBF produces good surface quality and dimensional accuracy in comparison with other AM methods; however, this process still suffers from a lack of dimensional accuracy and rough surface quality in comparison with conventional machining [5-7].

Ti-6Al-4 V has a high strength-to-weight ratio and is therefore popular in the aerospace, automotive and medical industries. The mechanical properties, microstructure and melt pool features of LB-PBF Ti-6Al-4 V components are thoroughly discussed in the literature [8-10]. However, another prominent factor, which has as yet received little attention, is the surface quality of the LB-PBF components. The average surface roughness $\left(R_{a}\right)$ achieved by this process is approximately 15-20 times higher than for conventional machining methods such as milling and grinding. This compromised surface finish of parts can cause high mechanical frictional forces and premature failure of parts during operation due to surface-initiated cracks [11-13]. It is well known that increasing the production rate in LB-PBF negatively affects the surface quality and mechanical properties. de Formanoir et al. [14] showed that by increasing the layer thickness from 30 to $90 \mu \mathrm{m}$, the roughness on the vertical and horizontal surface increases from 4.32 to $15.24 \mu \mathrm{m}$ and 6.22 to $10.13 \mu \mathrm{m}$ respectively, which can increase the probability of failure under mechanical loading. Khorasani et al. [15] proposed a model to predict the average areal surface roughness $(\mathrm{Sa})$ and analysed the effect of laser power, scan speed, hatch space and scan pattern angle on the quality of the surface of LB-PBF of Ti-6Al-4 parts. They showed that the process parameters drive the rheology of meltpool and surface quality. This study proved that heat treatment can also affect the surface quality of the printed component. Gibson et al. [16] showed that a conventional multi-axis process can improve both dimensional accuracy and surface quality of the LB-PBF curved parts. However, due to the brittle nature of LB-PBF parts, the chattering effect from cutting tools was observed, thus reducing the surface quality.

Abrasive machining is broadly used in the industry for polishing, deburring, finishing and other purposes. The biggest advantages of this process are its simple operation, no need for expert operators and no constraint of the sample shape or clamp-free (jig and fixtures). Besides, almost all materials can be processed with this technique [17-19]. Ferchow et al. [20] investigated abrasive flow machining for polishing internal surfaces of LB-PBF tubular components. This study showed that an acceptable deviation was found between the proposed rheological model and the measurements for material removal in a thickness of down-skin and up-skin areas. Chemical-assisted magnetic abrasive finishing of Inconel 625 tubes was investigated by Singh et al. [21]. The effect of process time, rotational speed, abrasive size, amount of abrasive and chemical agent on the surface quality and material removal rate (MRR) of Inconel parts was investigated in this study. Results showed that processing time and media size were the most influential parameters. Khorasani et al. [22] investigated the effect of $\mathrm{CBF}$ on solid components of stainless steel 316L made by LB-PBF. This study showed that surface roughness and MRR have a non-linear correlation with the process parameters, with an almost homogeneous material removal rate obtained by $\mathrm{CBF}$, making it a promising postprocess candidate to finish the surfaces of the LB-PBF parts.

$\mathrm{Na}$ et al. [23] worked on the effect of speed and time in abrasive centrifugal barrel finishing (ACBF) on the roughness of stainless steel parts. They found that surface roughness decreased by $70 \%$ at $60 \mathrm{rpm}$, and no significant improvement was observed for longer process times. Lesyk et al. [24, 25] compared ACBF, ultra-sonic impact treatment and ultra-sonic shot peening for polishing of Inconel 718 parts. The results showed that ACBF due to low impacting effect induced less residual stress compared to other methods. They also found that surface quality was enhanced by ACBF, while shot peening improved mechanical properties because induced compressive residual stresses improve micro and macro defects. Nalli et al. [26] worked on the effect of process parameters of ACBF on dimensions and tensile behaviours of $\mathrm{Ti}-6 \mathrm{Al}-4 \mathrm{~V}$ parts. The results showed that the surface quality improved by increasing rotational speed while time was found as another important factor in this process by Boschato et al. [27].

Most of the current studies, however, relate to either conventional machining or advanced material removal processes for simple LB-PBF geometries and external surfaces. The surface conditions of internal LB-PBF surfaces have not been considered. This research, based on Taguchi L18 samples printed by an LB-PBF process, provides detailed analysis of the improvement to the surface quality for both internal and external surfaces, where centrifugal barrel finishing (CBF) was used as post-processing. Different 
properties such as hardness and machinability, including surface roughness, material removal rate and dimensional accuracy, were examined to characterize the effect of CBF on LB-PBF components. This timely research will provide the field with useful knowledge about this commercially significant post-processing technique.

\section{Experimental setup}

\subsection{DoE}

In the case of using full-factorial DoE, the number of samples, production time and cost of the experiments increase for expensive experiments such as in AM processes. In this experiment, we used Taguchi DoE to reduce the cost and time of the experiment that have an insignificant influence on the accuracy of the results compared to full-factorial DoE [28, 29]. Taguchi L18 DoE was selected to analyse four parameters on different levels. The examining factors are speed direction, rotational speed, rotational time and volumetric percentage of abrasive media. The proposed DoE factors in each column have to be examined independently so that the number of replications in each column is balanced, and the design is stated as orthogonal. Before the main test set, the initial experimentations at different speeds, time, and volumetric percentage of abrasive were carried out and to find out the best values for selecting the optimum process parameters. Table 1 shows the process parameters and their levels. For the used machine, two types of rotational speed including clockwise $(\mathrm{CW})$ and counter clockwise (CCW) were available. In addition, three levels for rotational speed, time and the volumetric percentage of abrasive fluid were selected; therefore, the (L18: $2^{1} \times 3^{3}$ ) matrix was selected for DoE.

\subsection{Powder material and powder bed fusion process}

Samples were printed using an SLM 125HL system (SLM Solutions GmbH, Lubeck, Germany), equipped with YLRFiber laser. The samples were manufactured in bulk including engineered hollow features and then wire cut from the

Table 1 Experimental process parameters and levels

\begin{tabular}{llll}
\hline $\begin{array}{l}\text { Rotational } \\
\text { direction }\end{array}$ & $\begin{array}{l}\text { Rotational } \\
\text { speed (RPM) }\end{array}$ & $\begin{array}{l}\text { Rotational } \\
\text { time }(\mathbf{m i n})\end{array}$ & $\begin{array}{l}\text { Volumetric percentage } \\
\text { of abrasive fluid }(\%)\end{array}$ \\
\hline CW & 100 & 150 & 60 \\
CCW & 125 & 180 & 70 \\
& 150 & 210 & 80 \\
\hline
\end{tabular}

solid bed to the nominated sizes. Table 2 shows the process parameters of this experiment.

The powder material was Ti-6Al- $4 \mathrm{~V}$, and the specimens were designed in the form of a hollow component with a combination of circular, hexagonal, and square hollow features (Fig. 1). The size of the hollow feature for each sample was designed in such a way that provides the same hollow cross-sectional area and volume for each feature, which was done to remove any effect of different surface-to-volume ratios. The result is to have a fair comparison between the different hollow geometry. The hollow part specimens were designed to investigate how increasing internal curvature changes the MRR. The lowest curvature is given by the continuous curve of the circular while the mid-level curvature is represented by the hexagonal with an angle of $60^{\circ}$, and the highest-level curvature is represented by the square hollow (right angle corner).

\subsection{Abrasive centrifugal barrel finishing process}

CBF uses media, compound and water to surface finish and deburrs components [30, 31]. Centrifugal action causes a very fast, highly controllable operation. $\mathrm{CBF}$ is also called mass orbital barrel finishing and maintains a smooth rubbing action with little damage on the components, making it possible to produce high surface quality for both ductile and fragile parts.

A commercial MFI HZ-40 from Mass Finishing Incorporation equipped with Latched-End Barrel was used for mass finishing of hollow LB-PBF samples. For Ti-6Al-4 V and the size of the samples, the original equipment manufacturer (OEM) recommended equilateral triangle media $2 \times 2 \mathrm{~mm}$ from BV products, which are shown in Fig. 2A. To perform the post-processing water, media and printed parts were loaded in latched-end barrel with a length and diameter of $317.5 \times 174.6 \mathrm{~mm}$, respectively. The process parameters were selected based on OEM recommendations. The control factors identified in the Taguchi L18 DOE (Table 1) were applied to quantify the effect on internal and external hardness and roughness as well as MRR of the hollow area.

Table 2 Machine build process parameters

\begin{tabular}{ll}
\hline System parameters & Value \\
\hline Min. scan line/wall thickness & $120 \mu \mathrm{m}$ \\
Operational beam focus variable & $100 \mu \mathrm{m}$ \\
Layer thickness & $30 \mu \mathrm{m}$ \\
Laser power & $175 \mathrm{~W}$ \\
Hatch space & $120 \mu \mathrm{m}$ \\
Scan speed & $560 \mathrm{~mm} / \mathrm{s}$ \\
Laser spot diameter & $200 \mu \mathrm{m}$ \\
\hline
\end{tabular}


Fig. 1 A Specimen plan view drawing (dimensions are in $\mathrm{mm})$. B Printed sample and halved sections

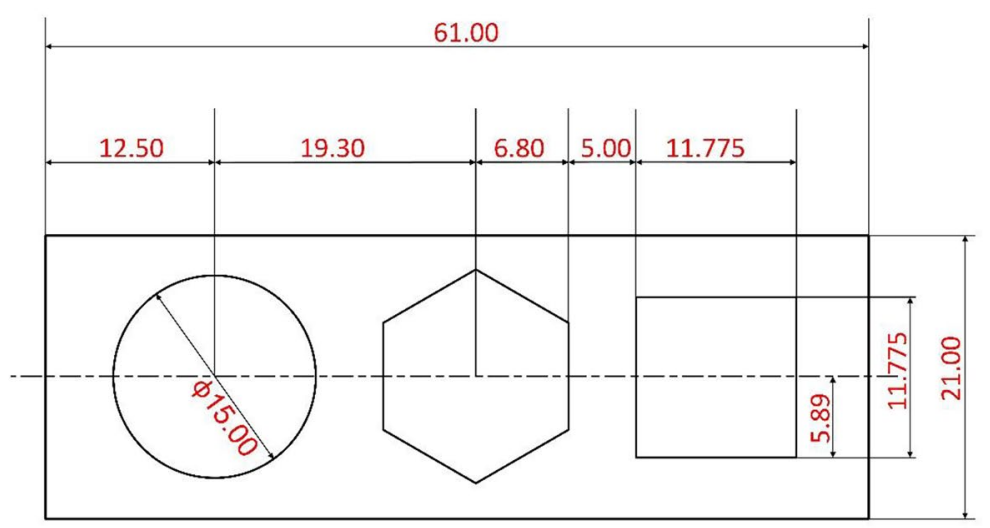

(A)

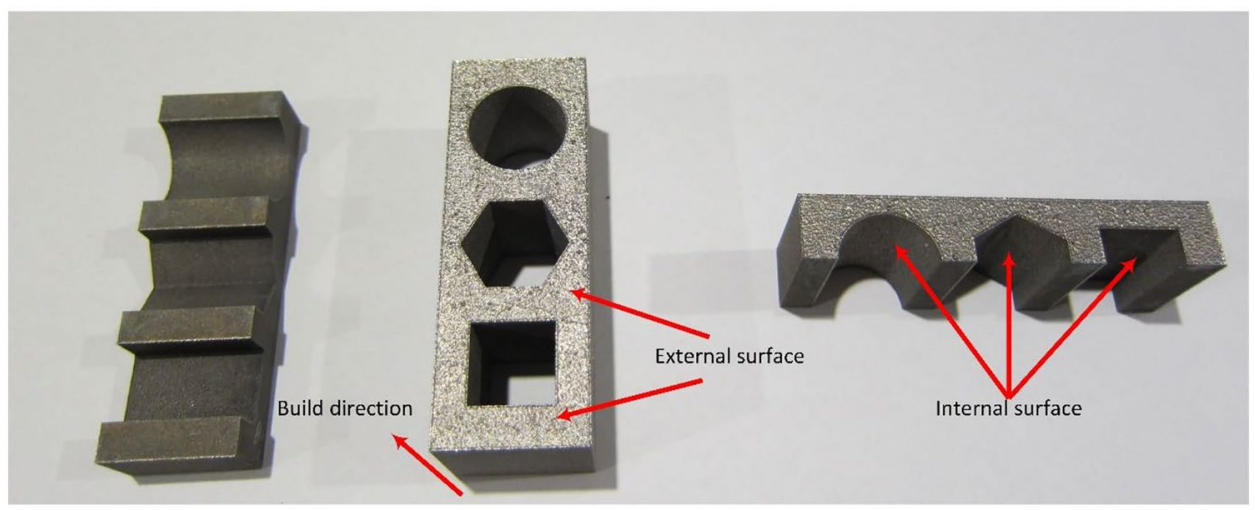

(B)

\section{Experimental procedure}

\subsection{Profilometry for surface roughness}

Surface roughness measurements were carried out by using Alicona Infinite Focus optical profilometer. For this purpose, the roughness of the samples before and after undergoing CBF was measured on both the outer and inner surfaces. The roughness of the sample was recorded in $R_{a}$,
$R_{q}$ and $R_{z}$, along with the direction of the laser. To obtain general and precise results, an area of $10 \times 10 \mathrm{~mm}$ was scanned for each measurement. The normalized value of surface parameters was calculated according to ISO 11056 and ISO 4288, and to filter the noise, a high-pass built-in Gaussian filter was used. The vertical and lateral resolutions of the profilometer were 400 and $10 \mathrm{~nm}$ respectively. The scanned area was selected randomly in different parts of the components. The outer measurements were carried

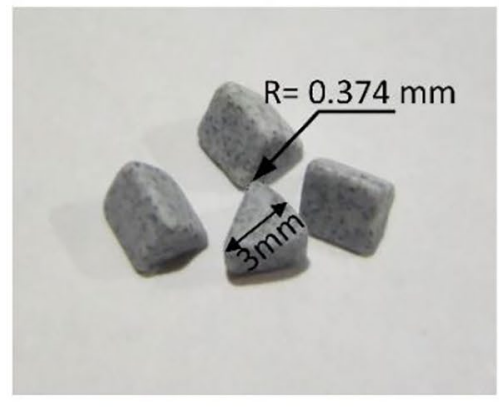

(A)

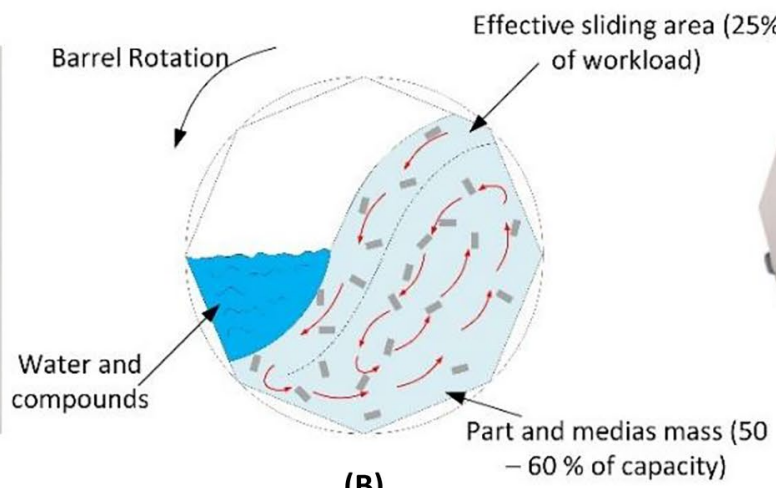

(B)

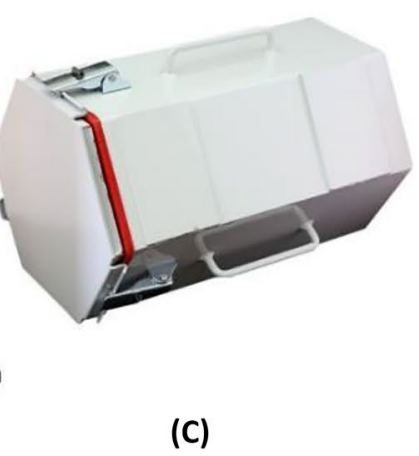

Fig. 2 A Media. B ACBF machine. C Latched-end barrel 
out in front and side of the samples. For the inside measurements, samples were wire cut in half to have access to internal surfaces.

\subsection{Dimensions, hardness and MRR}

The dimensions and deviations from the nominal values were measured by an optical measurement gauge. Samples were scanned vertically, and internal surfaces were captured and measured. In order to remove the effects of the attached powder particles and related errors, the samples were cleaned using an ultrasonic bath for $30 \mathrm{~min}$. To increase the repeatability and precision, each measurement was repeated five times. Distance measurements were conducted for the square hollow: all sides and the perimeter; for the hexagonal hollow: all diameters and the perimeter and the circular hollow: two diameters and the perimeter. Then, we used the side length for the square and the diameter for the hexagonal and circular for the calculations. Figure 3 shows the measurements.

Struers Durajet Hardness Tester was used for harness measurements in the Brinell scale. For the as-received and machined samples, hardness was measured at 20 points on the external surface, and the average values were reported. In addition, after post-processing, samples were sectioned by electrical discharge wire, and the hardness of internal surfaces was measured. For each hollow geometry, 10 points were measured from each sectioned piece.

\subsection{Microstructure characterization}

The overall microstructure of the CBF Ti-6Al-4 V alloys was investigated on the cross-section containing both internal and external surfaces as indicated in Fig. 12. The samples were hot mounted, ground with 4000 grit $\mathrm{SiC}$ paper then mechanically polished with $0.4 \mu \mathrm{m}$ OPS suspension. The electron backscattered diffraction (EBSD) analyses were conducted in a FEG Quanta 3-D FEI scanning electron microscope (SEM) under a $20 \mathrm{kV}$ and $8 \mathrm{nA}$ probe condition. Areas of $100 \times 100 \mu \mathrm{m}$ were mapped with a step size of $0.2 \mu \mathrm{m}$. The post-processing of the EBSD maps was accomplished using the TexSEM Laboratories Inc. software (TSL OIM analysis $6 \times 64$ ).

\section{Results}

\subsection{Taguchi analyses}

To verify the performance of the experimental results based on Taguchi DoE, "signal to noise ratio" (SNR) diagrams were generated (Fig. 4). SNR shows the control factors that can decrease the variability in an experiment by minimizing the effects of uncontrollable factors (noise factors). In this section, we show the SNR for the experimental variables, which are obtained from internal surfaces such as MRR and average roughness. To provide a more robust mechanical assembly with tighter tolerances, higher surface quality is required. Therefore, the criterion of "smaller is better" was selected for this factor. Meanwhile, for MRR, the criterion of "larger is better" was selected to increase the processing speed and reduce the cost of production. In this type of analysis, the more horizontal SNR curves identify the lower influence of the input parameters on the variability of the outputs [32]. Higher values of the SNR classify control factor settings that minimise the effects of the noise factors. As can be seen in Fig. 4, the effective variables for MRR are rotational speed and volumetric percentage of abrasive. For the internal $R_{a}$ of hexagon, SNR shows that the only effective factor is the volumetric percentage of abrasive; however, for the circular and square hollows, a volumetric percentage of abrasive was detected as an effective factor. The SNR also shows that the third and first levels of rotational speed and

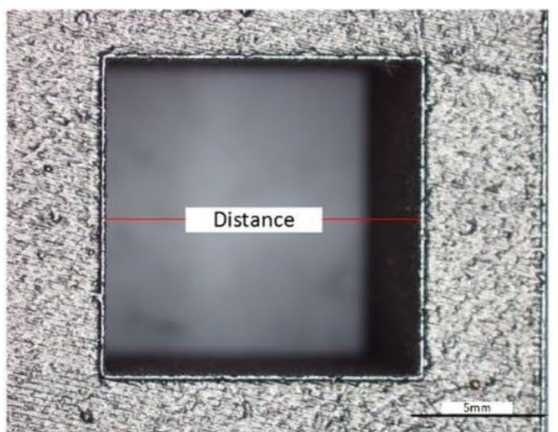

(A)

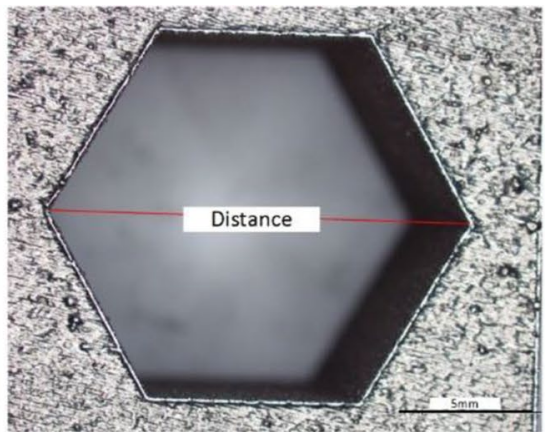

(B)

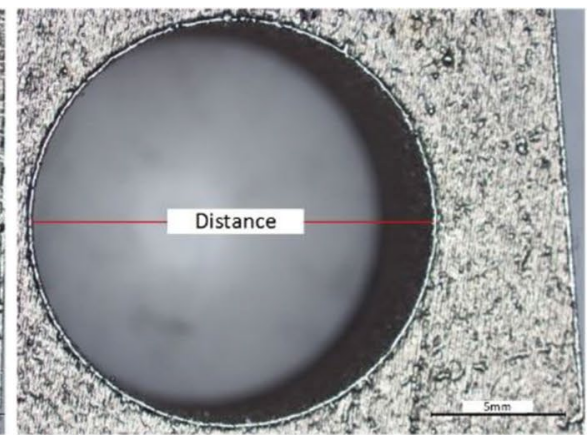

(C)

Fig. 3 Dimensional measurements by optical device A square, B hexagonal and $\mathbf{C}$ circular hollow features 

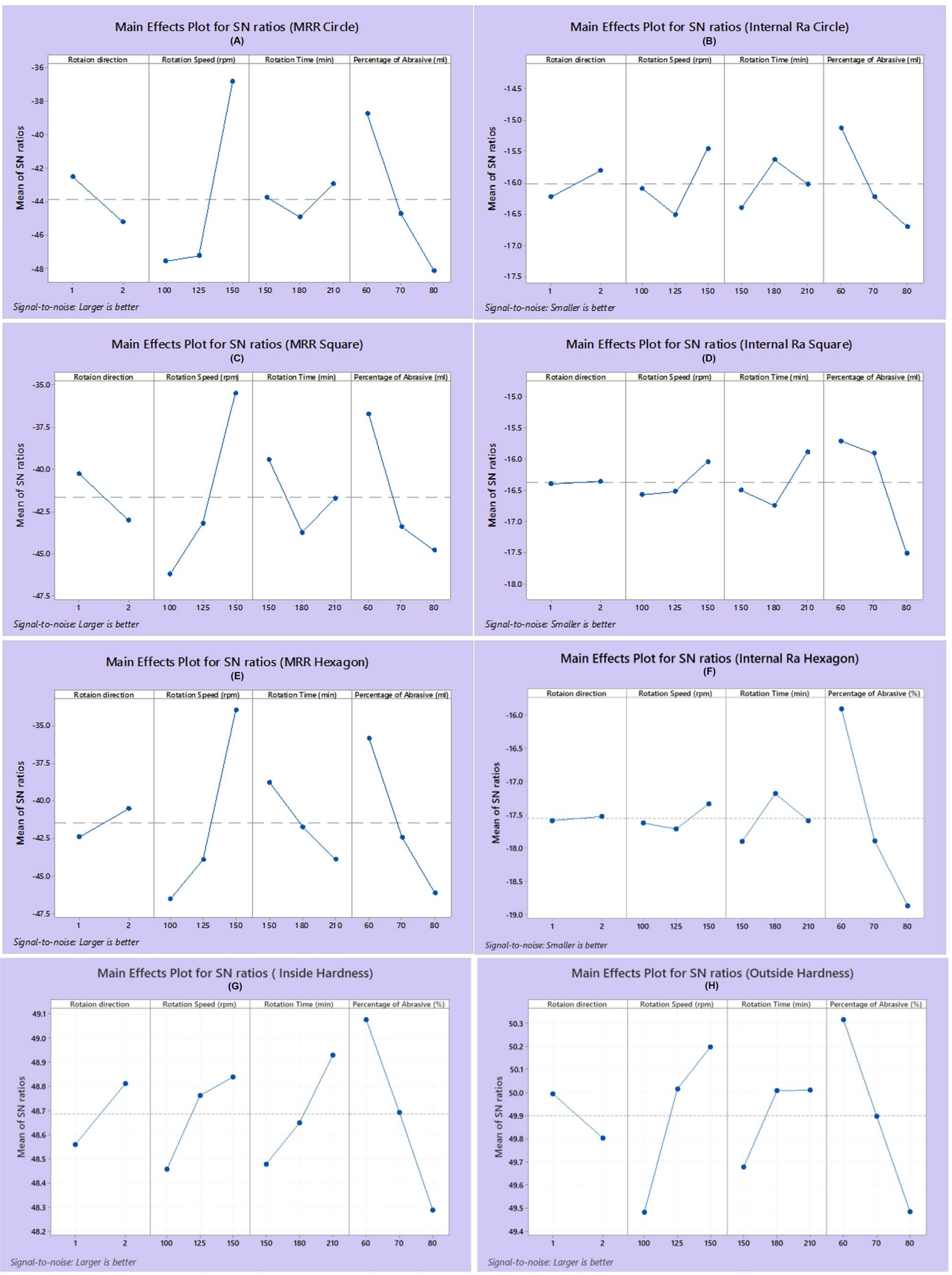
४Fig. 4 Signal-to-noise and mean analysis A, B MRR and internal $R_{a}$ for the circular, C, D MRR and internal $R_{a}$ for square MRR and internal $R_{a}$ for the hexagonal and $\mathbf{G}, \mathbf{H}$ internal and external hardness ( 1 is related to clockwise barrel rotation and 2 is related to counter clockwise barrel rotation)

volumetric percentage of abrasive respectively provide the appropriate conditions to reduce variability in the roughness and increase the MRR.

\subsection{Analysing the effective parameters using MANOVA}

Multivariate analysis of variance (MANOVA) is a statistical method to compare the multivariate average of outputs. This analysis can be used considering that more than one dependent variable influence the results. In a MANOVA, statistical differences on one continuous dependent factor by an independent grouping factor are analysed. The results of MANOVA will show whether or not the independent grouping variable (inputs) has a significant effect on the dependent variable or outputs $[33,34]$. The significance of the dependent variables can be determined by comparing them to the null hypothesis using $P$-values. The null hypothesis proves (to a given confidence level) that no relationship between the two investigated variables exists (that is, the input variable does not affect the output). The level of statistical significance is expressed as a $P$-value between 0 and 1 . To accept or reject the null hypothesis, the value of the $P$-value is calculated. A smaller $P$-value proves stronger evidence to reject the null hypothesis. In this analysis, $P \leq 0.05$ shows that the independent variable can significantly change the dependent variable, which in turn shows strong evidence against the null hypothesis. It means that there is less than a 5\% probability that the null hypothesis is correct. The $P$-value analysis has been carried out, and the results based on Wilk's criterion are listed in Table 3. Table 3 shows the value of $\mathrm{MRR}, R_{a}$ and hardness.

According to the results, the volumetric percentage of abrasive fluid was found to have a $P$-value of $\leq 0.05$ for almost all dependent variables or outputs. Also, for most of the experiments, the mean analysis showed that the processing speed has a $P$-value of $\leq 0.05$. Thus for these two factors, the null hypothesis is rejected. The volumetric percentage of abrasive and rotational speed has a significant impact on the variation of external hardness, MRR and external roughness. Interestingly, the results showed that the internal surface roughness of the square and circular has no effective process parameters. In addition, the internal hardness and internal surface roughness of the hexagonal have only a single effective process parameter. The results of MANOVA and SNR analysis confirm that rotational direction and processing time have a negligible effect on almost all of the dependent variables because $P$-values $>0.05$, and therefore, the null hypothesis is accepted for these variables. Thus, the outputs are not changed significantly by changing these two parameters.

\section{Discussion}

From the $P$-value analysis, the effect of the rotational speed, time and the percentage of media on the outputs were found and will be now discussed.

\subsection{Interaction of effective parameters on the roughness and MRR for hollow areas}

Figure $6 \mathrm{~A}-\mathrm{C}$ shows that the rotational speed and the percentage of the abrasive are significant variables tochange MRR. Figure 6A-C shows that, in this experiment, the MRR increases by increasing the rotationalspeed.

The highest value of MRR and the lowest roughness were obtained when the barrel was loaded to about $60 \%$ of capacity with a mixture of workpiece, media, water and compounds. Higher load levels to $80 \%$ reduce the length of the slide, thus decreasing the chance of contact between the workpiece and media. This leads to a reduction in media wear and a reduction of the force of workpiece contact and subsequently MRR. The media wear or depreciation rate of the media is calculated according to Eq. (1):

$M_{w}=\frac{\left[1-\left(\frac{f}{i}\right)^{3}\right] \times 100}{H}$

where $f$ is the final dimension of media pieces, $i$ is the initial dimension of media pieces, and $H$ is working time. Since the rotation of media is completely stochastic, the wear rate for each side and direction are assumed to be the same. To calculate the media wear, the sizes of 50 media after each test were measured, and the average values were used in Eq. (1). The values of final media dimensions and media wear rate for different loading values are presented in Table 4 .

As shown in Fig. 5, by using higher loads the media wear reduces. These results prove that higher barrel loading causes less contact, tool wear and therefore less material removal for hollow components.

To analyse the effect of rotational speed, process time and the volumetric percentage of abrasive media, main effect diagrams for each of the dependent variables were plotted.

The results showed that the internal roughness of circular and square hollows is not significantly changed by the variation of process parameters as shown in Fig. 6D, E. The results of the material removal showed that for all circular, square and hexagonal hollow shapes, rotational speed and the volumetric percentage of abrasives have a significant 
Table $3 P$-values from a statistical test for MANOVA

\begin{tabular}{|c|c|c|c|c|c|c|c|c|}
\hline & \multicolumn{4}{|l|}{ MRR circular } & \multicolumn{4}{|l|}{ MRR square } \\
\hline & $\begin{array}{r}\text { Rotational } \\
\text { direction }\end{array}$ & $\begin{array}{l}\text { Rotational } \\
\text { speed }\end{array}$ & Process time & $\begin{array}{l}\text { Abrasive fluid } \\
(\%)\end{array}$ & $\begin{array}{r}\text { Rotational } \\
\text { direction }\end{array}$ & $\begin{array}{l}\text { Rotational } \\
\text { speed }\end{array}$ & Process time & $\begin{array}{l}\text { Abrasive fluid } \\
(\%)\end{array}$ \\
\hline \multirow[t]{3}{*}{$P$-value } & 0.678 & 0.025 & 0.92 & 0.049 & 0.466 & 0.0008 & 0.267 & 0.0004 \\
\hline & \multicolumn{4}{|c|}{ MRR hexagonal } & \multicolumn{4}{|c|}{ Internal $R_{a}$ (circular) } \\
\hline & $\begin{array}{r}\text { Rotational } \\
\text { direction }\end{array}$ & $\begin{array}{l}\text { Rotational } \\
\text { speed }\end{array}$ & Process time & $\begin{array}{l}\text { Abrasive fluid } \\
(\%)\end{array}$ & $\begin{array}{r}\text { Rotational } \\
\text { direction }\end{array}$ & $\begin{array}{l}\text { Rotational } \\
\text { speed }\end{array}$ & Process time & $\begin{array}{l}\text { Abrasive fluid } \\
(\%)\end{array}$ \\
\hline \multirow[t]{3}{*}{$P$-value } & 0.54 & 0.001 & 0.495 & 0.009 & 0.587 & 0.473 & 0.687 & 0.210 \\
\hline & \multicolumn{4}{|c|}{ Internal $R_{a}$ (square) } & \multicolumn{4}{|c|}{ Internal $R_{a}$ (hexagonal) } \\
\hline & $\begin{array}{l}\text { Rotational } \\
\text { Direction }\end{array}$ & $\begin{array}{l}\text { Rotational } \\
\text { Speed }\end{array}$ & Process Time & $\begin{array}{l}\text { Abrasive Fluid } \\
(\%)\end{array}$ & $\begin{array}{l}\text { Rotational } \\
\text { Direction }\end{array}$ & $\begin{array}{l}\text { Rotational } \\
\text { Speed }\end{array}$ & Process Time & $\begin{array}{l}\text { Abrasive fluid } \\
(\%)\end{array}$ \\
\hline \multirow[t]{3}{*}{$P$-value } & 0.894 & 0.711 & 0.63 & 0.113 & 0.932 & 0.962 & 0.648 & 0.011 \\
\hline & \multicolumn{4}{|c|}{ External $R_{a}$ (front surface) } & \multicolumn{4}{|c|}{ Internal hardness } \\
\hline & $\begin{array}{r}\text { Rotational } \\
\text { direction }\end{array}$ & $\begin{array}{l}\text { Rotational } \\
\text { speed }\end{array}$ & Process time & $\begin{array}{l}\text { Abrasive fluid } \\
(\%)\end{array}$ & $\begin{array}{r}\text { Rotational } \\
\text { direction }\end{array}$ & $\begin{array}{l}\text { Rotational } \\
\text { speed }\end{array}$ & Process time & $\begin{array}{l}\text { Abrasive fluid } \\
(\%)\end{array}$ \\
\hline \multirow[t]{3}{*}{$P$-value } & 0.472 & 0.0004 & 0.273 & 0.02 & 0.159 & 0.193 & 0.128 & 0.010 \\
\hline & \multicolumn{8}{|c|}{ External hardness } \\
\hline & $\begin{array}{r}\text { Rotational } \\
\text { direction }\end{array}$ & $\begin{array}{l}\text { Rotational } \\
\text { speed }\end{array}$ & Process time & $\begin{array}{l}\text { Abrasive fluid } \\
(\%)\end{array}$ & & & & \\
\hline$P$-value & 0.36 & 0.033 & 0.305 & 0.017 & & & & \\
\hline
\end{tabular}

$P$-values less than 0.05 have a significant effect

effect on the MRR. However, these variables do not significantly change the value of roughness, which is related to the random movement of the media. In mass finishing especially in rotary-based systems, the flow of media on the samples is not in the specific direction; therefore, it can remove the material in different directions ( $\mathrm{X}, \mathrm{Y}$ and $\mathrm{Z}$ ). The interaction of this random material removal process can affect the roughness. As a result, no significant change in the roughness of circular and square hollows is observed while MRR is significantly changed by rotational speed and the volumetric percentage of abrasives.

Up to a certain point, the faster the barrel rotates, the steeper the angle of the slide which results in higher removal of material in CBF. The best MRR and the most lustrous finish occur at a higher sliding velocity. In CBF, the sliding velocity is obtained by the following equation:
$V=\frac{N \times \pi \times D}{1000}$

where $N$ is rotational speed, and $D$ (in $\mathrm{mm}$ ) is barrel diameter. According to Eq. (2), increase in rotational speed or barrel diameter leads to an increase in velocity, which means higher centrifugal force applied to media and parts and as shown in Fig. 7 affects sliding. During barrel rotation, the abrasive particles inside the barrel form two types of layers, which are named the active layer and the passive layer. The thickness of the active layer depends upon the rotational speed and size of the abrasive particles. Rotational speed also drives the number of particles in the active layer [35]. Figure 7A shows the sliding path angle that appears for a low speed (around $55 \mathrm{~m} / \mathrm{min}$ that was obtained from Eq. (2)). Higher rotational speed increases the slope of the sliding
Table 4 Results of final media dimensions and media wear

\begin{tabular}{lllllr}
\hline Test no. & $\begin{array}{l}\text { Final media dimensions } \\
(\mathbf{m m})\end{array}$ & $\begin{array}{l}\text { Media wear } \\
\text { rate }(\%)\end{array}$ & Test no. & $\begin{array}{l}\text { Final media dimensions } \\
(\mathbf{m m})\end{array}$ & $\begin{array}{l}\text { Media } \\
\text { wear rate } \\
(\%)\end{array}$ \\
\hline 1 & 2.72 & & & & 7.60 \\
2 & 2.86 & 25.13 & 10 & 2.92 & 13.77 \\
3 & 2.97 & 13.72 & 11 & 2.85 & 9.11 \\
4 & 2.45 & 2.56 & 12 & 2.92 & 13.56 \\
5 & 2.90 & 45.02 & 13 & 2.86 & 9.89 \\
6 & 2.98 & 9.42 & 14 & 2.90 & 46.99 \\
7 & 2.50 & 1.49 & 15 & 2.43 & 40.21 \\
8 & 2.60 & 41.75 & 16 & 2.53 & 54.94 \\
9 & 2.55 & 34.98 & 17 & 2.30 & 38.11 \\
\hline
\end{tabular}



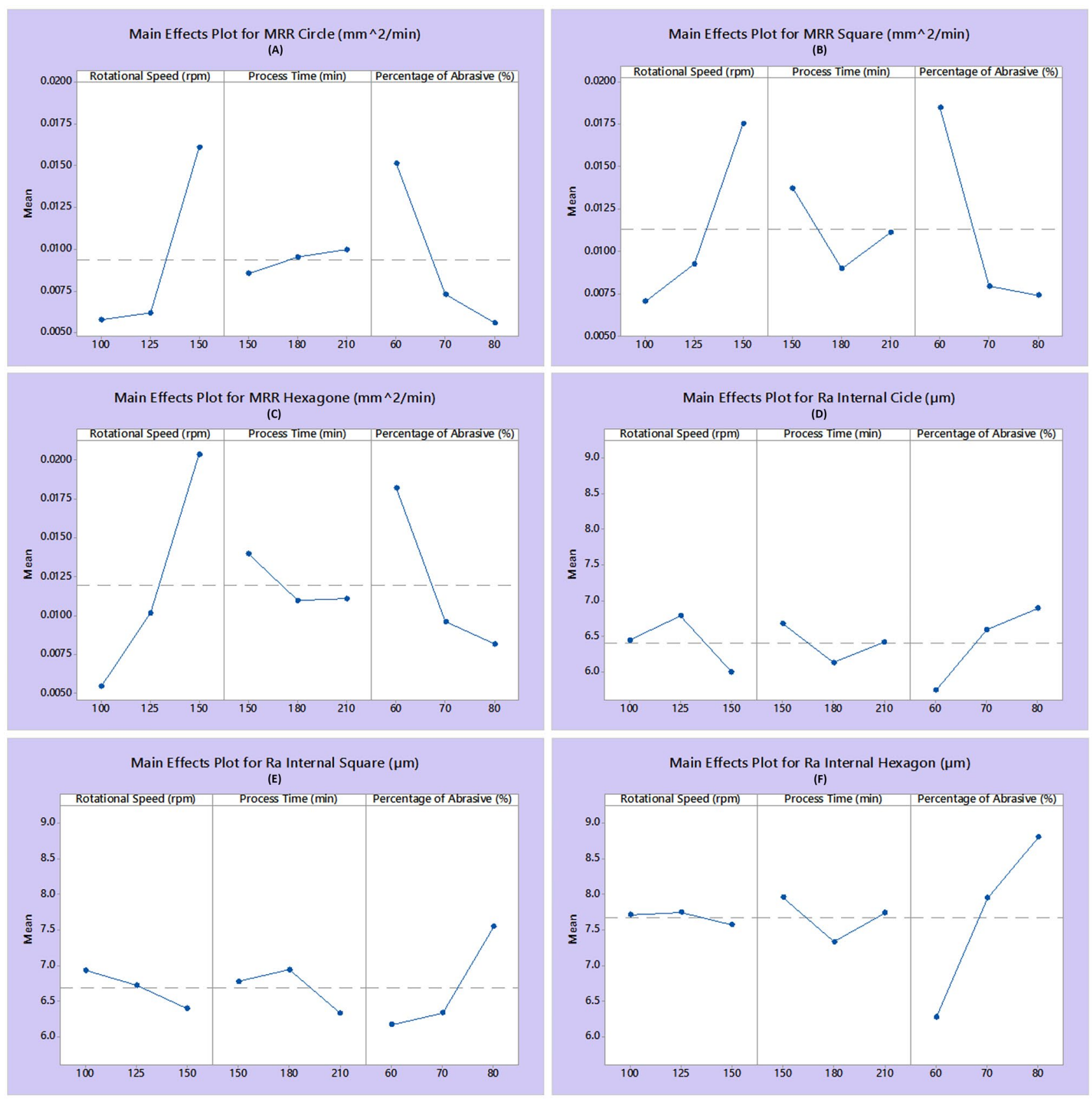

Fig. 5 Main effect plots for MRR and roughness

path (Fig. 7B, C), and in this condition, the sliding speed between abrasive media and the components increases. This leads to higher cutting or grinding operation which produces a higher MRR and media wear rate. Figure 7D shows how when the speed is higher than the optimum value and a pocket in the middle of the media is formed, the components are allowed to tumble against each other and cause damage or breakage. Figure 7E illustrates how when the rotational speed is extremely high, the centrifugal force pushes the media and the components to the walls of the barrel. In this condition, no contact happens between media and the components, so there is a radical reduction in the MRR. Since no damage occurred in our experiments, it is assumed that the first two cases occurred at the selected rotational speeds.

Figure $8 \mathrm{~A}$ shows the value of sliding velocity, media wear and MRR for different rotational speeds. In thisfigure, the black and red zones are related to the sliding layer of media. As can be seen in this figure, slidingvelocity increases by increasing the rotational speed. The deburring or cutdowncut down operations areperformed better at higher 


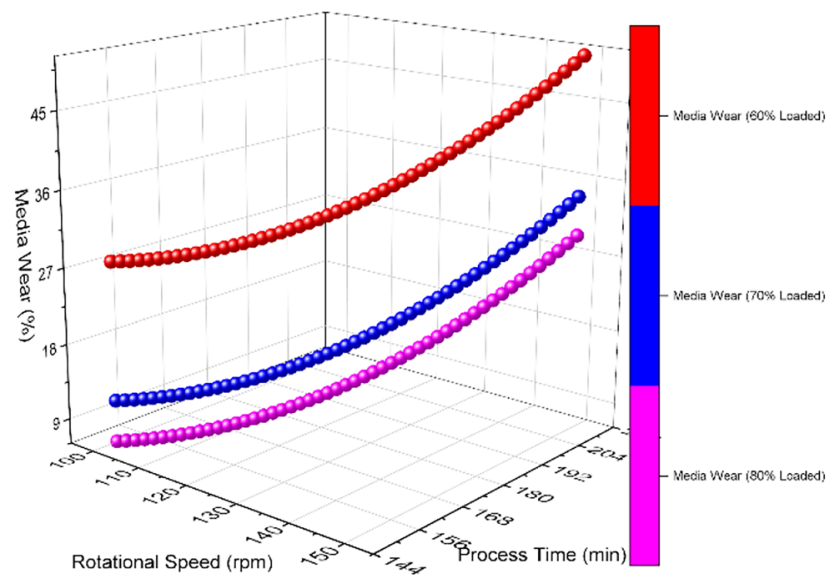

(A)

Fig. 6 A Media wear and B MRR for different loadings

speeds. Figure 8B shows that in higher rotational speeds, more media wear isobserved. The media wear is directly related to material removal rate; therefore, when rotational speed reaches $150 \mathrm{rpm}$, the slope of $\beta 3$ increases drastically (Fig. 7C), leading to a significant increase in media wear rateand MRR. As a result, a substantial increase in MRR and media wear are is observed for a rotational speed of 150 rpm. Table 5 in the appendix Appendix shows the roughness values for the reference sample, and Tables 6, 7 and 8 represent MRR, hardness and surface roughness for all test cases. Figure 7 and theresults of Fig. 8 show that the operational mode for our test cases was similar to the presented conditions in Fig. 7A-C.

\subsection{The effect of hollow shape on area of removed materials and MRR}

To find out the effect of hollow shapes on the mass finishing process, the value of MRR and area of removed materials is investigated. Figure 8C, D show that the area of removed material and MRR is a direct function of hollow shape. The MRR in the circle is lower than hexagon and square. It is related to the difference between contact areas of abrasive media in different shapes. In the firDeclast point, it is necessary to mention that the size of media is less than holes in all shapes, so, media can easily flow in the holes. Contact mechanism in a circular hole is almost point to point and liner also may happen rarely, and the flat surfaces of media have no effects on material-removing (surface to surface contact cannot happen in this shape). But in the case of the flat surface of hexagonal and square shape, the contact mechanism is point to point for the corner of media, line to line for the edge of media and surface to surface for flat surfaces of media, so the area of contact between media and surface of the part in square and hexagonal shape is

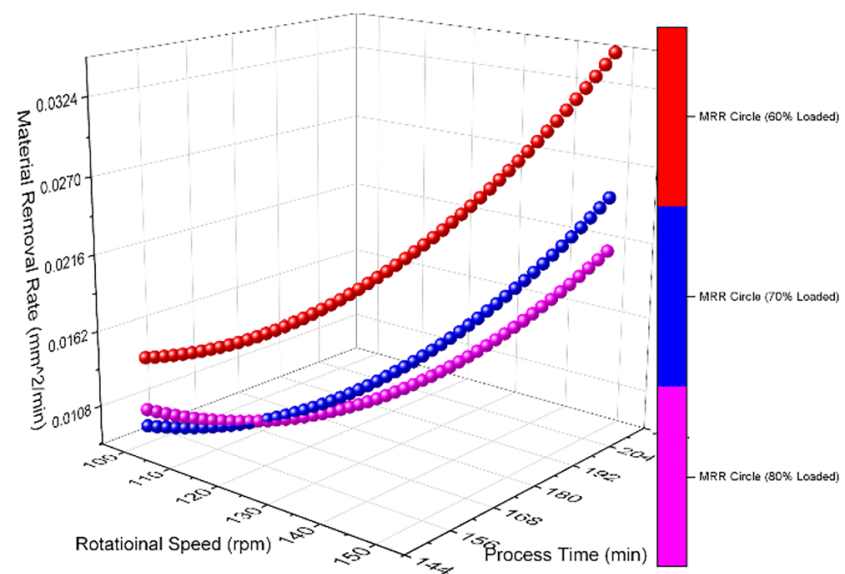

(B)

extremely more than circular shape. The highest dimensional deviation and MRR were found for the hexagonal hollow followed by the square and circular hollow geometry. The optical measurements (Fig. 3) show that this observation is related to the material removal rate in the corners. Rougher surfaces were found in the area close to the corners, which shows that CBF has a shortcoming when removing material from geometrically constrained areas. Figure 9A shows that the step height $\mathrm{H} 1$ is smaller than the media radius $R_{m}$. In this case, the media will often bend the burr over rather than cut/remove it, and complete removal needs a long cycle time. However, increasing the cycling time in mass finishing processes can thermally affect the media and the component, which finally results in breaking and impingement. As a result, less cutting occurs when $\mathrm{H} 1<R_{m}$ such as in the circular hollow geometry in our experiment. This leads to producing a smaller area of removed materials and MRR for circular hollows that is illustrated in Fig. 8C, D.

When samples have larger step height, as shown in Fig. 9B, the media can work over the edge and against both vertical and horizontal surfaces of the component, which can eliminate the above issue. Comparing the results of the area of removed materials and MRR for the hexagonal and square hollows shows that the CBF process is more effective for the hexagonal hollows. This is related to the geometrical contact of the media and the corners as well as the subsequent flow of the media in the hollow. When the inner surface is finished by CBF, the near fillet radii of the component $\left(R_{W}\right)$ must be equal to or exceed the major radius on the media $\left(R_{m}\right)$ (Fig. 10C). The mentioned ratio of the media and hollow radius allows the media to abrade easily on the corners and have a more efficient material removal process. Thus, hexagonal hollow shows to have a higher area of removed material and MRR. The media has an equilateral shape, and the corner of the media will better fit the fillet of the hollow 


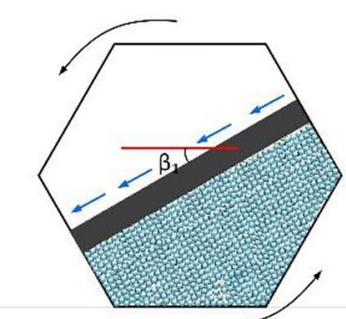

(A)

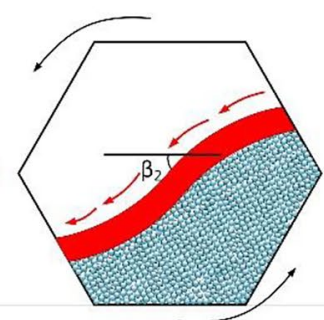

(B)

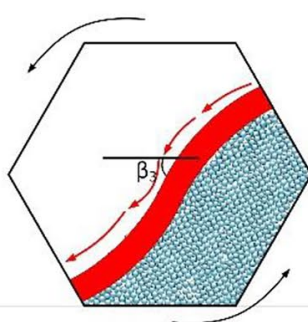

(C)

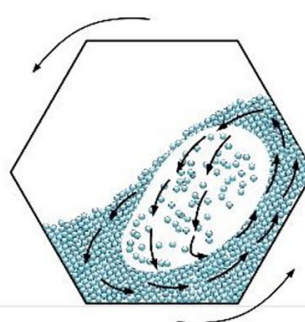

(D)

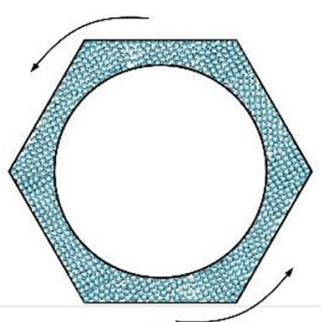

(E)

Fig. 7 Actions in barrels at different speeds, $\mathbf{A}$ low speed and sliding angle, B, C medium speed and sliding angle, D high speed and pocked in the middle, $\mathbf{E}$ extremely high speed and no machining ( $\beta$ is sliding angle)

hexagonal, a region with an angle of $\Theta=120^{\circ}$, while the media is unable to access the fillet area for the square corner (Fig. 10E, F). These two corner regions are untouched by the media and will not receive significant deburring action. The untouched region area of the hexagon and square was obtained by Eqs. (3) and (4). In this equation, $R$ is the radius of media corners:

Section area of hexagon $=R^{2}\left(\tan \left(\frac{\pi}{6}\right)-\frac{\pi}{6}\right)$

Section area of square $=R^{2}-\frac{1}{4} \pi R^{2}$
The radius of the media $R_{m}=0.365 \mathrm{~mm}$, so the values of the section area for hexagonal and square obtained were $0.0072 \mathrm{~mm}^{2}$ and $0.0286 \mathrm{~mm}^{2}$ respectively. This estimates that the untouched area for the hexagonal hollow is four times smaller than the square hollow, and this is another reason for the higher area of removed material and MRR for the hexagonal hollow shape.

When $H$ is significantly bigger than the radius of the media, such as for our square hollow shape, the reaction force of the component edges lifts the media (Fig. 10B) and the effective contact points slide on the edges and reduce the efficiency of the deburring process. However, for hexagonal, as shown in Fig. 10C, D, the media is rotating on the curves, and both effective contact points and effective surface (on
Fig. 8 A Sliding speed of different rotational speeds. B The persantage of media wear in diffrent barell time. C Material removal rate of hollow shapes in each rotational speed. D Average removed areas in each hallow shape
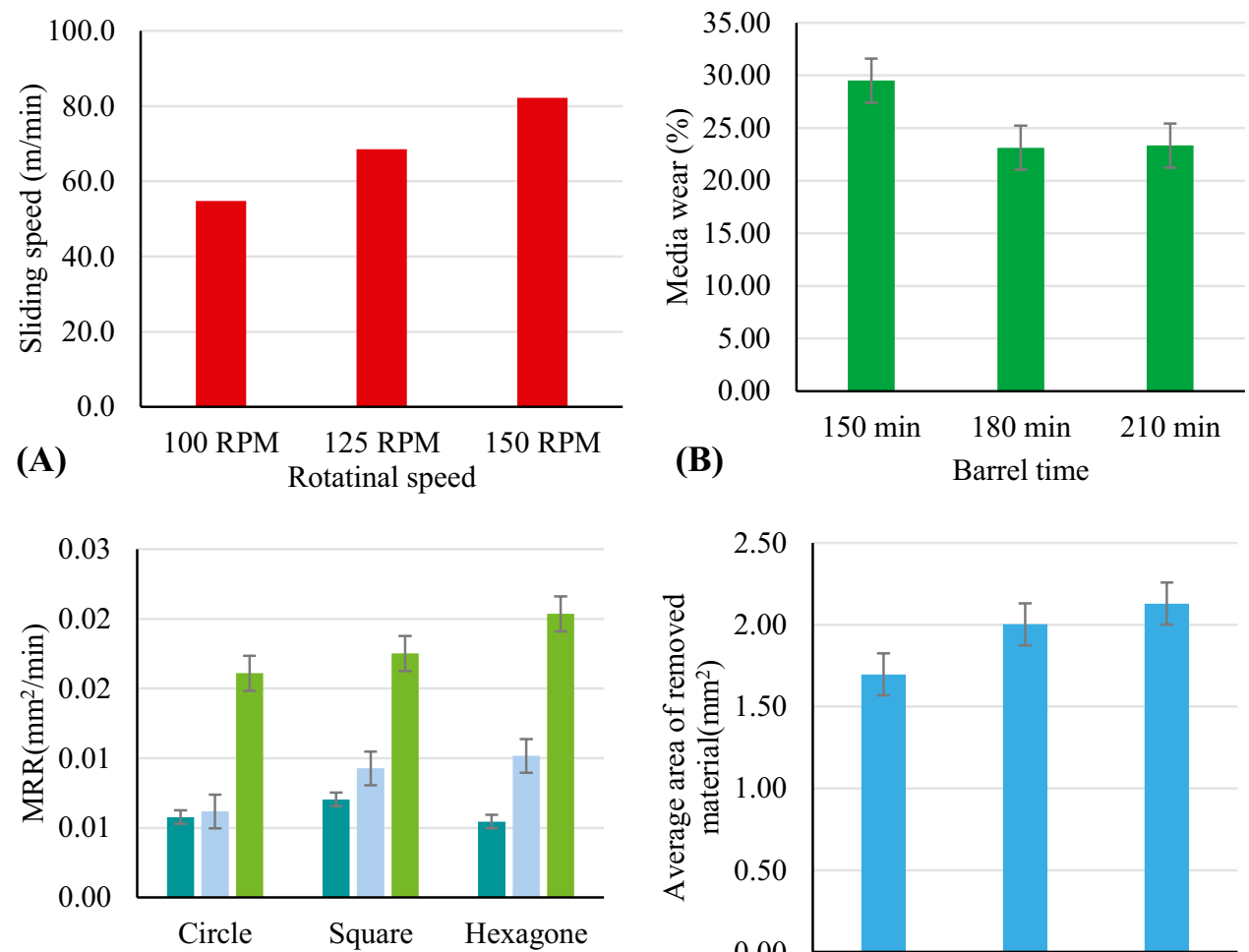

(C) $₫ 100$ RPM $\square 125$ RPM $\square 150$ RPM

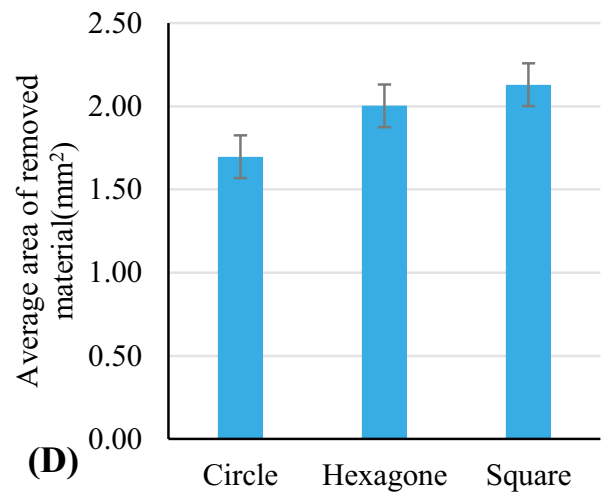




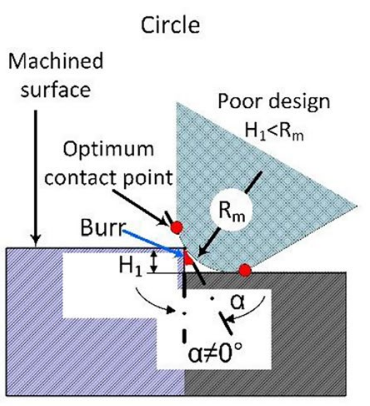

(A)

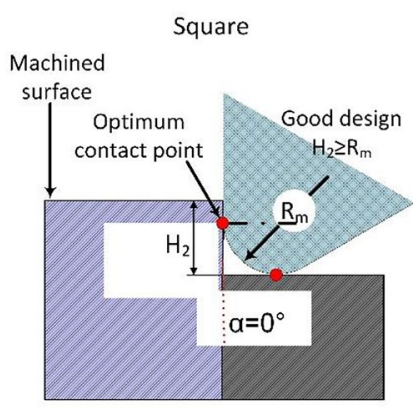

(B)
Fig. 9 A Poor design and deburring and $\mathbf{B}$ good design and complete deburring ( $\alpha$ is deburring angle)

the radius of the media) remove the material. In this case, the efficiency of the deburring improves, and the MRR and area of removed materials increase, which is in agreement with Fig. 8C, D.

\subsection{Interaction of effective parameters on the internal and external hardness}

Figure 11 shows the interaction between rotational speed, process time and the percentage of media on external roughness, internal and external hardness. As can be seen in Fig. 11, the roughness of external surfaces has a relative trend with the hardness. Also, the results of the $P$-value analysis in Table 3 and Fig. 6 show that the external roughness has an indirect relationship with the rotational speed and direct trend versus the volumetric percentage of abrasive media. Higher rotational speed increases the slope of the active area $(\beta)$ in the barrel (Fig. 8A-C), which produces
Fig. 10 Poor design for material removal A first step and $\mathbf{B}$ second step of the removal process. Good design $\mathbf{C}$ first step and D second step of the removal process

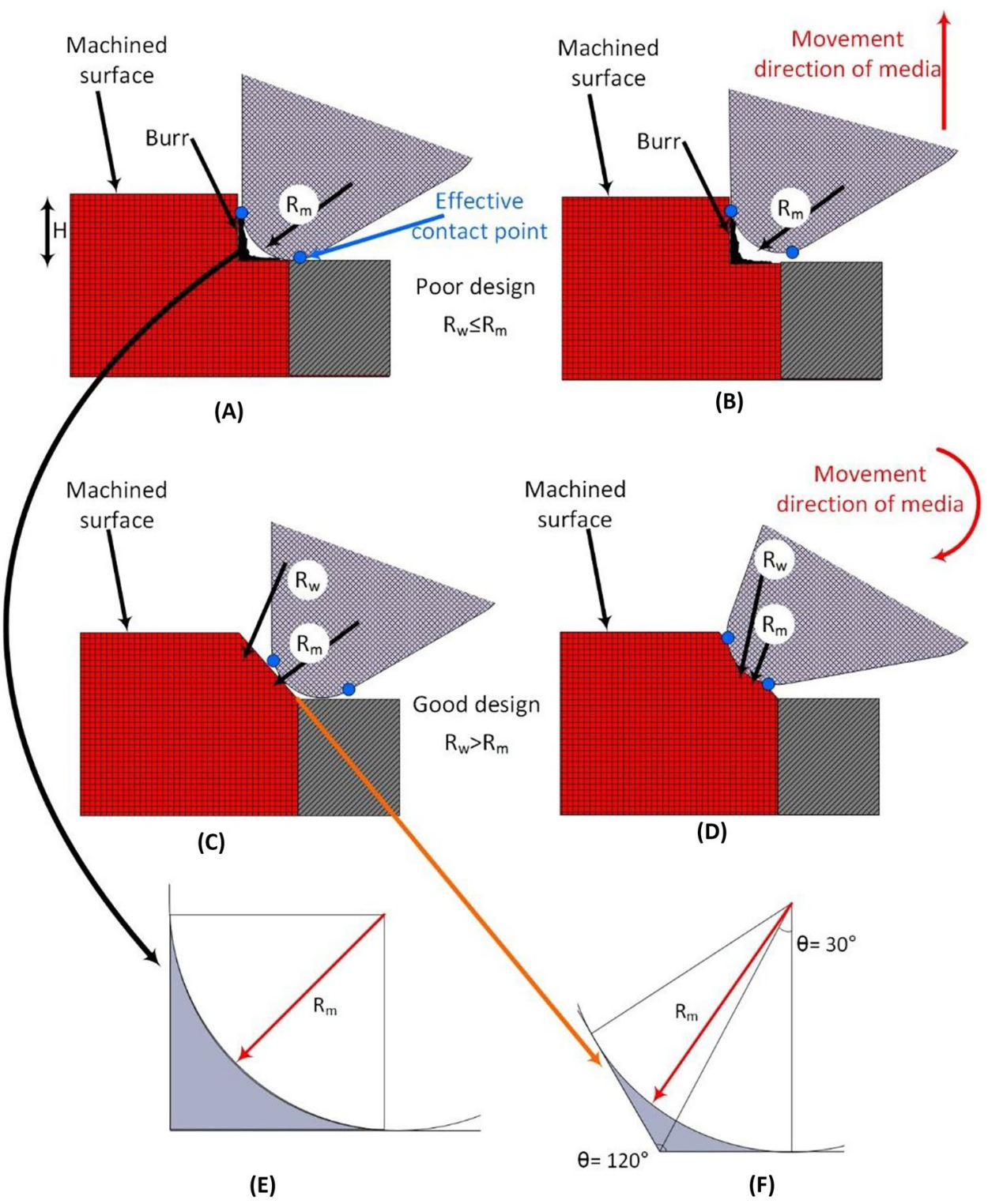



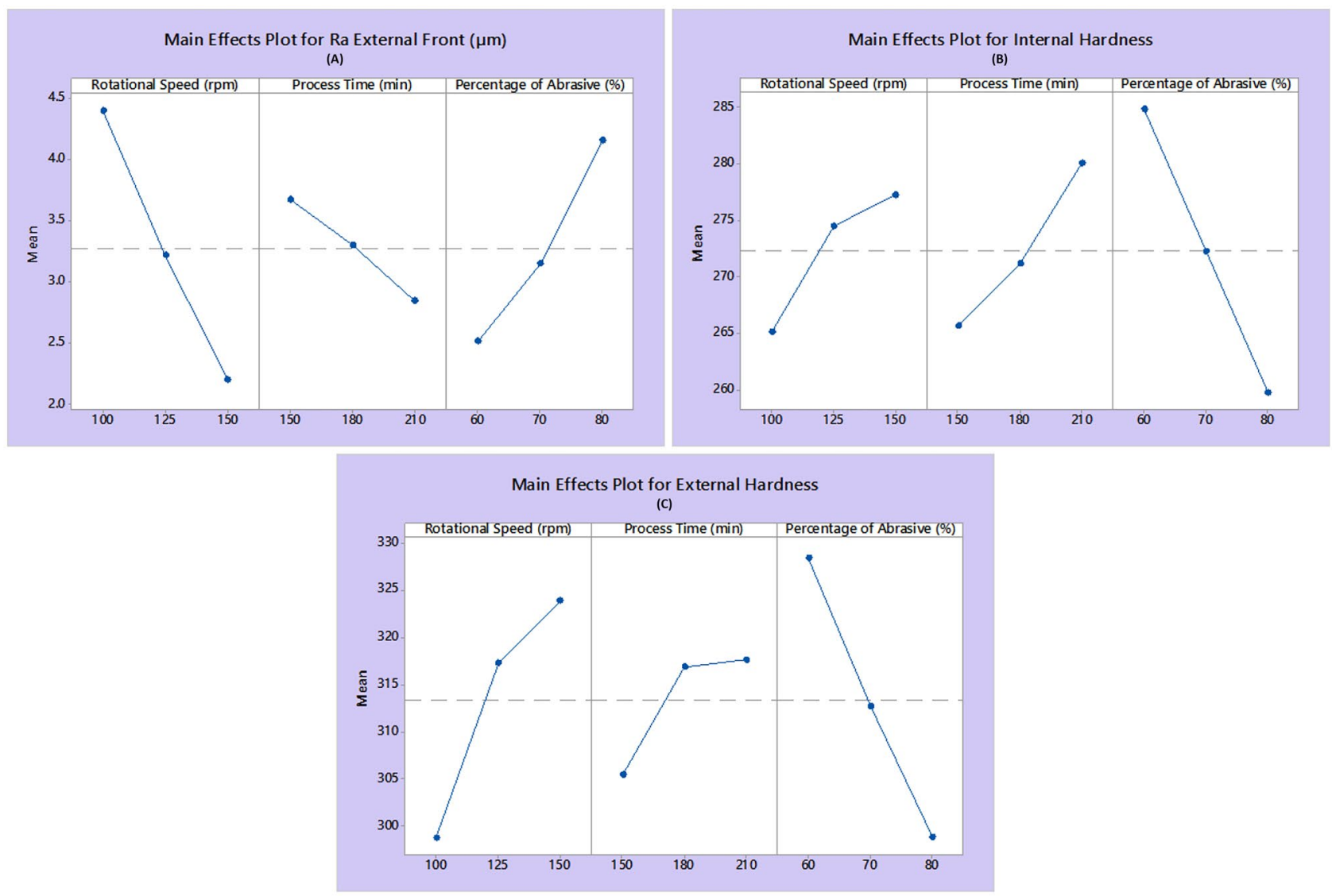

Fig. 11 Main effect plot for $\mathbf{A}$ external roughness $\mathbf{B}$ internal hardness and $\mathbf{C}$ external hardness

a higher sliding velocity (Fig. 8A). The higher the sliding velocity of the media, the higher the impact force on the component, so the wear rate for both media and the component increases, which leads to removal of the roughness and improvement in the surface quality. Figures 6 and 11 also show that the area of deviation and MRR is directly related to the external roughness. This proves that higher MRR produces the better surface quality of the external surfaces.

A lustrous surface finish in external surfaces was obtained using the latch-end barrel with a length and diameter of $317.5 \times 174.6 \mathrm{~mm}$ and the appropriate speed of $\frac{33}{\sqrt{D}}(D$ is the diameter of the barrel). As the rotational speed $N$ increases and approaches $\frac{49}{\sqrt{D}}$, the sliding velocity increases so that the cutting efficiency and MRR increases. Our primary test showed when the rotational speed is greater than $\frac{49}{\sqrt{D}}$ due to high centrifugal force, the relative motion stops between the burrs and the components, and no cutting occurs.

External surface roughness showed similar behaviour with internal surfaces against the barrel load. Figure 12 shows that by increasing the barrel load, the slope of the active line $(\beta)$ reduces at even higher speeds. Thus, the sliding speed reduces and media/component wear and MRR decrease, which is in agreement with the results presented in Fig. 5. In other words, the media flow in higher barrel loads reduces, which negatively affects the surface quality.

\subsection{Evaluation of the CBF processed $\mathrm{Ti}-6 \mathrm{Al}-4 \mathrm{~V}$ microstructure}

Similar to the other surface treatments such as cold rolling $[36,37]$, shot peening $[38,39]$ or laser shock peening [36, 40], during the CBF process, the surface region experiences severe plastic deformation, leading to significant stress concentrations, the formation of dislocation structures and possible grain refinement. According to the Hall-Petch theory (Eq. (5)), reducing grain size to $10 \mathrm{~nm}$ leads to an increase in the strength of the material. It is related to increasing the number of dislocation and sliding systems in material and increasing the resistance of lattices to motions of dislocation.

$\sigma=\sigma_{0}+\frac{k}{\sqrt{d}}$

In Eq. (5), $\sigma_{0}$ is a constant value for the stress and is related to the resistance of lattice to dislocation motions, $k$ 
Fig. 12 The slope of the active line in different barrel loadings and rotational speeds, A low rotational speed, $\mathbf{B}$ medium rotational speed, $\mathbf{C}$ high rotational speed for $60 \%$ abrasive fluid, D low rotational speed, E medium rotational speed, $\mathbf{F}$ high rotational speed for $70 \%$ abrasive fluid, $\mathbf{G}$ low rotational speed, $\mathbf{H}$ medium rotational speed, I high rotational speed for $80 \%$ abrasive fluid ( $\beta$ is sliding angle)

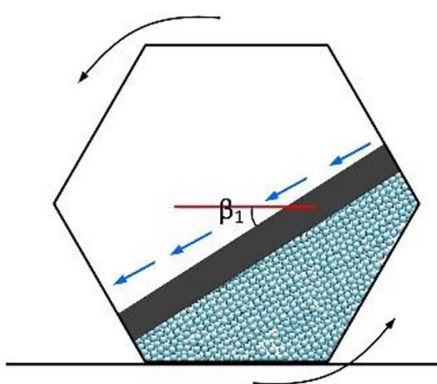

(A)

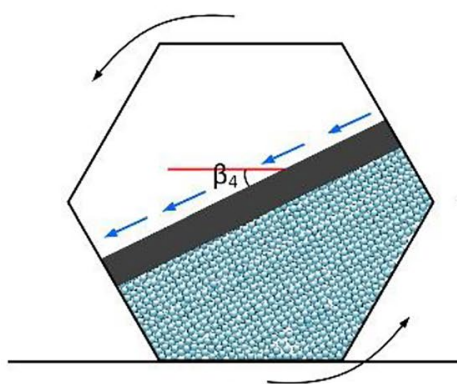

(D)

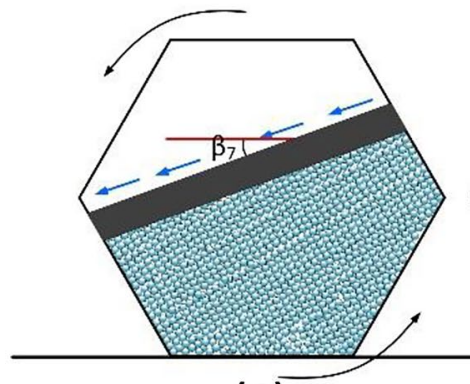

(G)

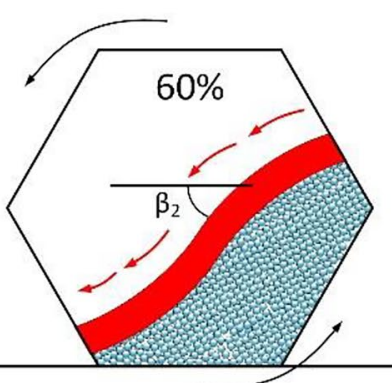

(B)

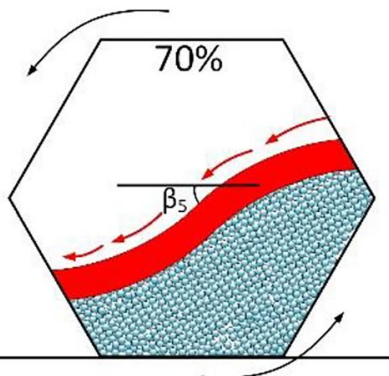

(E)

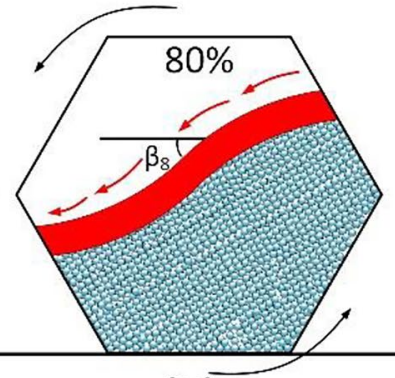

(H)

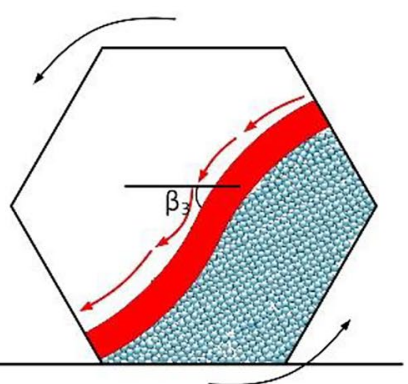

(C)

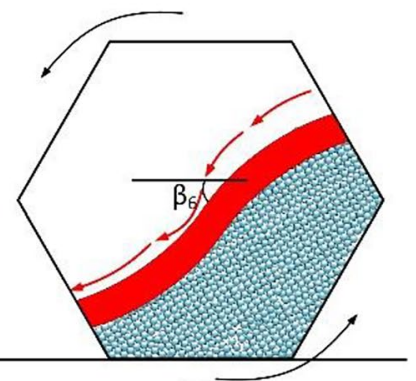

(F)

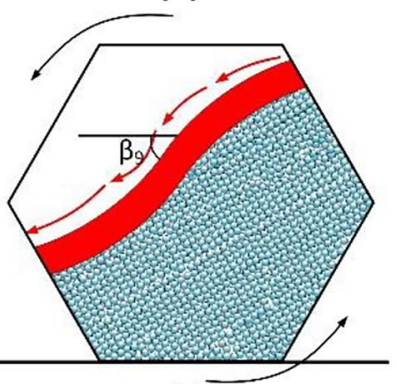

(I) is the constant specific coefficient for the material named strengthening factor, and $d$ is the average grain size.

This gradient of characteristic microstructure from the surface to the interior plays an integral role with regard to the mechanical and chemical performance of the material and hence requires extensive attention. To this end, the microstructure and crystallographic characteristics of the Ti-6Al-4 V alloy after the CBF process were investigated to analyse the evolution of microstructure for both the internal and external surfaces. Figure 13 reveals the orientation imaging embedded with image quality maps for the crosssection areas of test cases 9 and 10 (see Tables 6 and 8). These samples were selected to trace the microstructure characteristics after a high, 38\% (Sample 9), media wear rate and a low, 7\% (Sample 10), media wear rate as indicated in Table 4 . In both conditions, the cross-section shows a typical colony basket weave- $\alpha$ microstructure consisting of thin $\alpha$-laths in coarse prior $\beta$ grains separated continuous $\beta$ films. This is a typical observation of LB-PBF-produced Ti-6Al-4 V alloys where the later deposited layers experience lower cooling rates, resulting in the formation of a Widmanstätten-like $\alpha$ structure within the columnar $\beta$ grains along the build direction [41-43].

The Kernel Average misorientation (KAM) analysis was conducted to analyse the strain distribution on the surface region of the CBF samples (Fig. 14). KAM analysis can be used as a measure of local grain misorientation. KAM quantifies the average misorientation around a measurement point with respect to a defined set of nearest neighbour points. In this mode, the local misorientation assigned to the centre point of a particular grain with respect to all points in the perimeter of the kernel is measured. This map reveals the local variations of lattice orientations due to the presence of dislocations which therefore can be considered a manifestation of the residual strain. Generally, KAM is high in deformed grains due to higher dislocation density [44, 45]. The area near the surface representing higher KAM values (coloured region with green, yellow and red) is confined to a thin layer for all conditions, indicating a relaxed surface developed through the CBF procedure. This region can be 
(a)

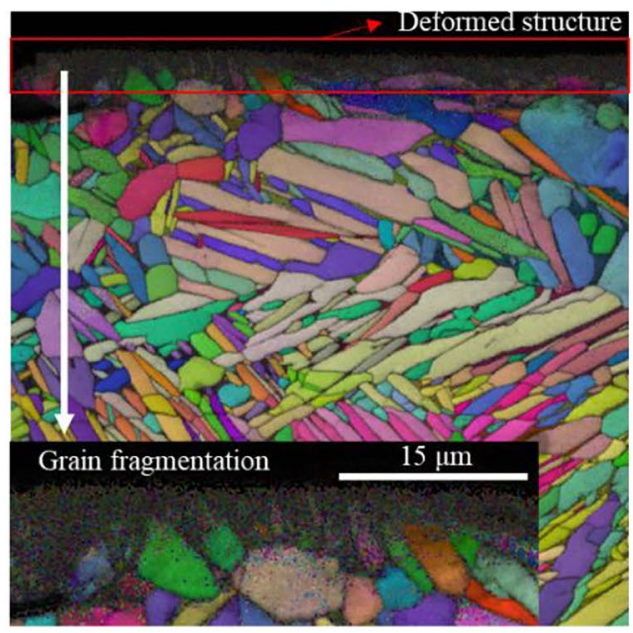

(c)

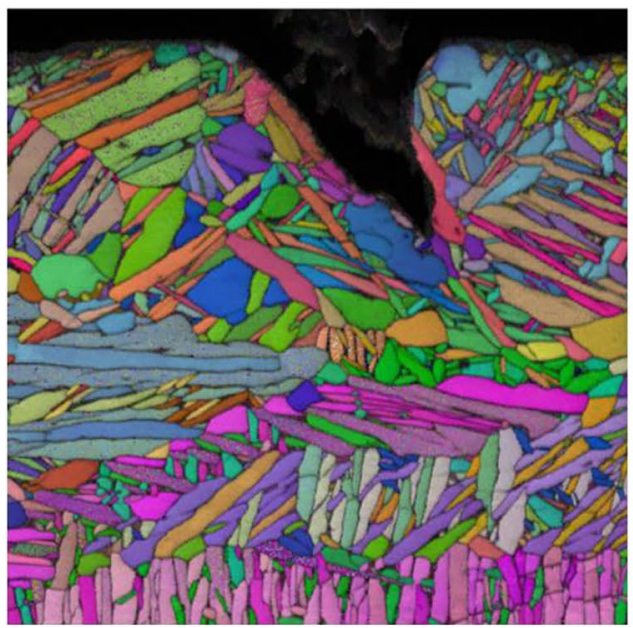

$40 \mu \mathrm{m}$ (b)
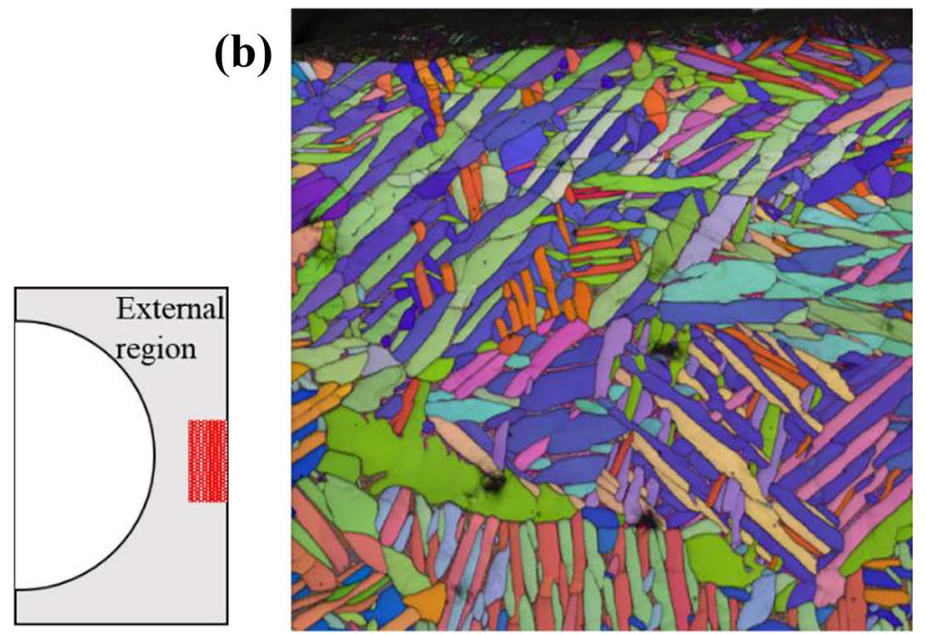

(d)
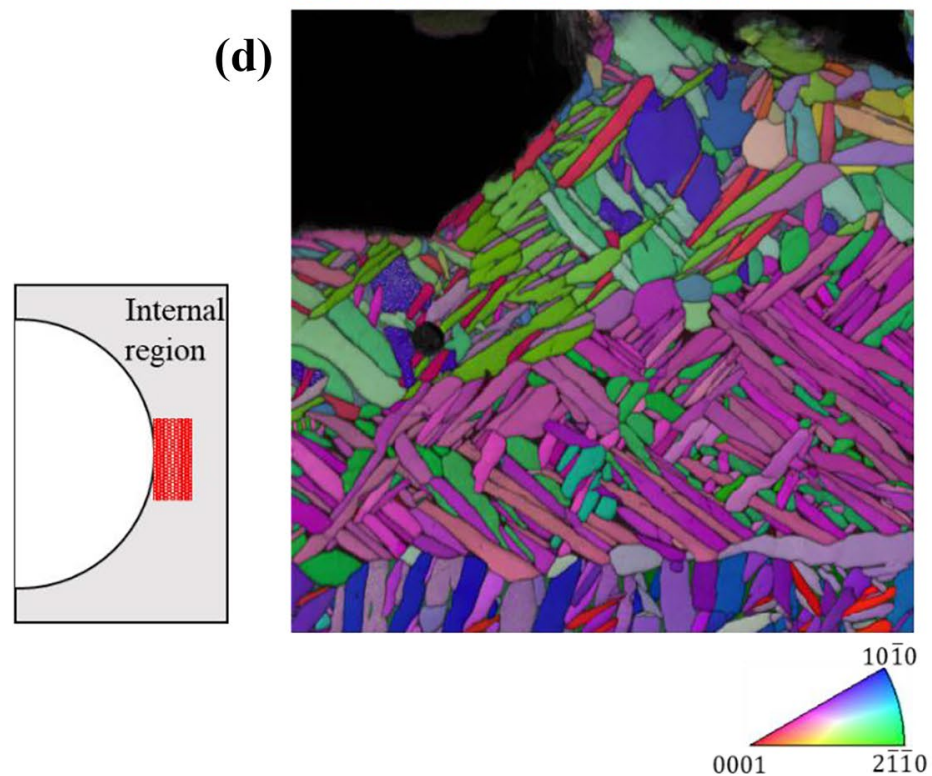

Fig. 13 The orientation maps embedded with the image quality maps for a sample 9 and b sample 10 external, $\mathbf{c}$ sample 9 and $\mathbf{d}$ sample 10 internal surfaces

also identified as lower indexed areas (darker regions) at the top of the KAM region (Fig. 14). The increase in the KAM values is associated with a higher local misorientation induced by the surface deformation of CBF media. Therefore, as the process becomes intense, a higher value of KAM and a larger affected surface depth are to be expected. The thickness of the highly strained region (coloured region) is higher at the external surfaces for both test cases 9 and 10 . However, the internal surfaces have a relatively lower stress concentration compared to the external regions. For the more intense barrel finishing condition (i.e., sample 9), the thickness of the highly strained region is around $10 \mu \mathrm{m}$ and $3.5 \mu \mathrm{m}$ for the external and internal surfaces, respectively. On the other hand, the thickness was calculated at around $2.8 \mu \mathrm{m}$ (external surface) and $1.5 \mu \mathrm{m}$ (Internal surface) for sample 10. Interestingly, no signs of deformation structures such as slip lines or the well-known (1012) deformation twinning were observed within the deeper regions of the printed samples. This further shows that the impact of the plastic deformation induced by the media was only limited to the surface region of the printed Ti-6Al-4 V alloys.

The small depth of residual stress development compared to the other surface modification techniques is interesting as the previously reported results on shot peening and/or laser shock peening research reveal comparatively thicker regions up to $2 \mathrm{~mm}$ depth from the surface area [40,46]. The highly misoriented regions illustrate the severe deformation on the surface of the samples leading to the disappearance of the Kikuchi lines for EBSD measurements. The area of regions grows with the increase of the rotational speed and time at 
(a)

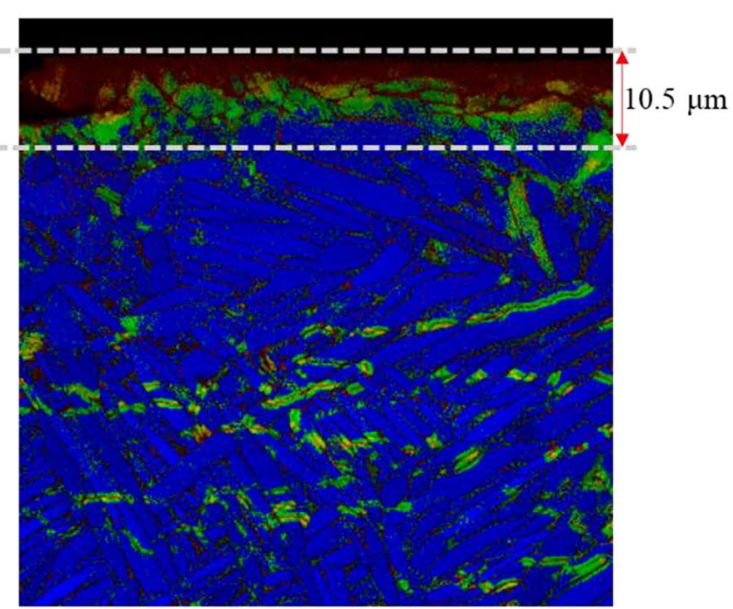

(c)

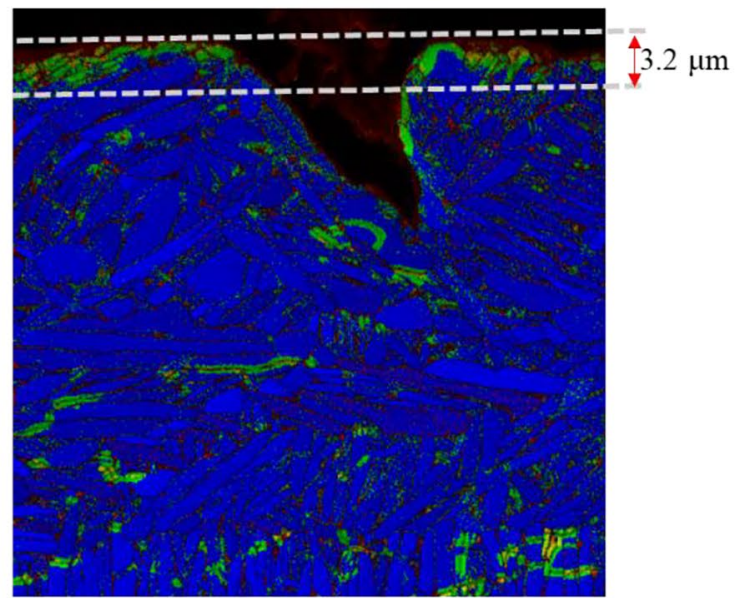

$40 \mu \mathrm{m}$ (b)

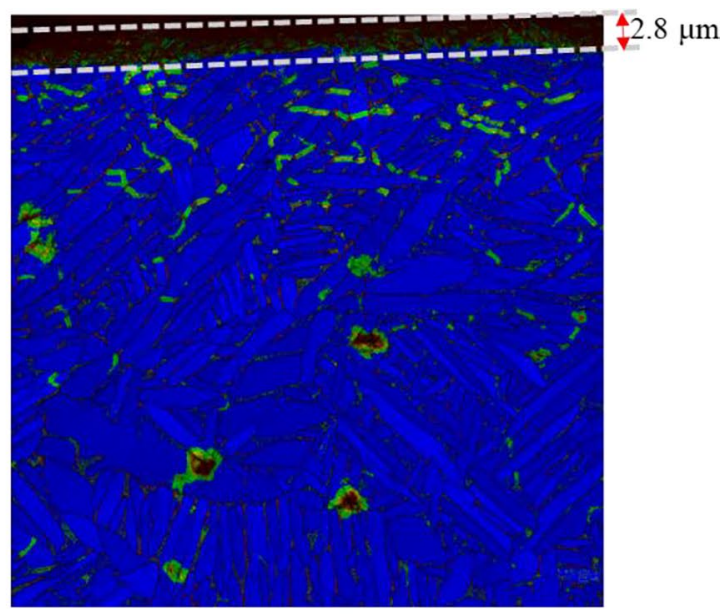

(d)

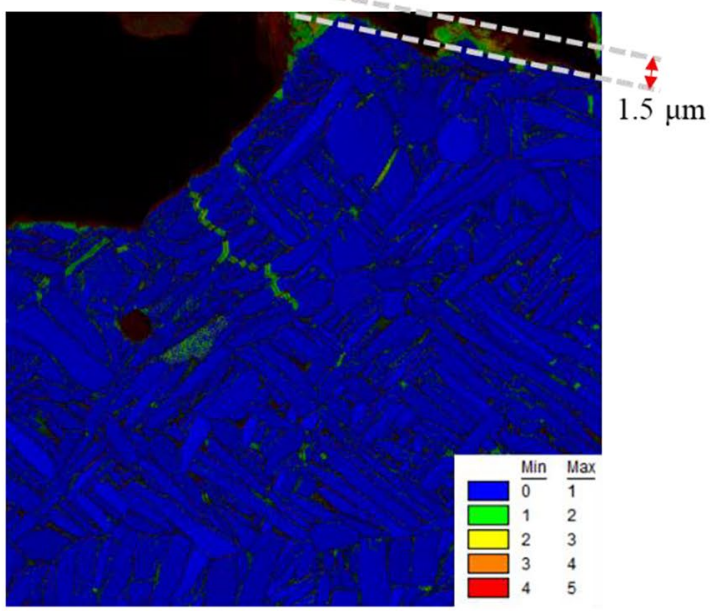

Fig. 14 The kernel average orientation maps embedded with the image quality maps for the a sample 9 and $\mathbf{b}$ sample 10 external, $\mathbf{c}$ sample 9 and d sample 10 internal surfaces

an optimum volumetric percentage of abrasive media. This increase in the deformed region can be observed within sample 9, which is further accompanied by the improvements in the surface hardness (in comparison to sample 10: Table 8). Furthermore, the current crystallographic orientation mapping reveals that deformation induced by the CBF can lead to fragmentation of the $\alpha$ laths into the refined structure (Fig. 13a). This highly refined region can inhibit the crack growth from the inner regions and effectively retard the premature component breakdown, leading to improvements in the fatigue life of the Ti-6Al-4 V alloy [38, 40]. Therefore, the CBF process may also play an important role in the printed material properties.

\section{Conclusion}

In this paper, the LB-PBF components were printed and post-processed by $\mathrm{CBF}$ to improve the internal and external surface quality. The effect of CBF process parameters was analysed on different dependent variables such as MRR, internal and external roughness and hardness. The effect of CBF process on the variation of residual stress and hardness was also identified from microstructure and crystallographic features. In $\mathrm{CBF}$, a few considerations for workpiece design should be taken into consideration to produce the best results. The tumbling media should have access to all surfaces and edges. Furthermore, the media must freely slide overall critical surfaces and edges; thus, the size of the media has to be selected in accordance with the area of the internal surfaces.

Results showed that the rotational speed and the percentage of the abrasive are the driving factors for MRR. The highest MRR and the best surface quality were obtained when the barrel was loaded up to $60 \%$ of volume with a mixture of water and compounds, workpiece and media. The length of the slide reduces in higher barrel loads up to $80 \%$. This reduces the chance of contact between media and workpiece, which leads to a reduction in contact force, media wear and MRR. 
The results showed that the internal roughness of circular and square hollows is not significantly driven by process parameters that can be related to the random movement of the media.

The results showed the CBF has a better performance for the external surfaces compared to the internal hollow area. This can be related to easier sliding on corner-free external surfaces.

The faster barrel rotation produces a steeper sliding angle and higher removal of material, so the best MRR and the most lustrous finish are obtained at higher sliding velocity. A higher rotational speed increases the slope of the sliding path and the sliding speed between abrasive media and component increases, which leads to a higher cutting or grinding process, MRR and media wear rate. When too high rotational speed is selected, the pocket in the middle of the media is shaped and tumbles the parts against each other, causing damage or breaking. In the case of extremely high rotational speed, centrifugal force sticks the media and the parts to the walls of the barrel, and no contact occurs between media and the parts so media wear and MRR drastically decreased.

Both MRR and the area of removed materials are a direct function of hollow shape. The biggest dimensional deviation and MRR were found for hexagonal hollow followed by square and circular, which can be related to the material removal process in the corners.

The best lustrous or surface finish for external surfaces was obtained when using a latch-end barrel with a length and diameter of $317.5 \times 174.6 \mathrm{~mm}$ and the rotational speed of $\frac{33}{\sqrt{D}}(D$ is the diameter of the barrel). As the rotational speed increases to $\frac{49}{\sqrt{D}}$, the sliding velocity increases; thus, the cutting efficiency and MRR also increase.

For an optimum CBF set of process parameters, a thick layer of deformed microstructure was obtained. The increase in the deformed layer depth was associated with an increase in the hardness of the CBF samples. This resulted from the small grain size structure along with high dislocation density development during the CBF process that may ultimately improve the crack propagation resistance and ultimately increase the fatigue life of Ti-6Al-4 V components.

\section{Appendix}

Table 5 Reference values

\begin{tabular}{lcc}
\hline Parameter & Value & Unit \\
\hline Internal $R_{a}$ for circular reference sample & 18.621 & $\mu \mathrm{m}$ \\
Internal $R_{a}$ for hexagon reference sample & 27.839 & $\mu \mathrm{m}$ \\
Internal $R_{a}$ for square reference sample & 20.249 & $\mu \mathrm{m}$ \\
External $R_{a}$ for reference sample & 21.098 & $\mu \mathrm{m}$ \\
Internal hardness & 222.40 & $\mathrm{HV}$ \\
External hardness & 256.09 & $\mathrm{HV}$ \\
\hline
\end{tabular}

Table 6 MRR for different hollow shapes

\begin{tabular}{|c|c|c|c|c|c|c|c|}
\hline Test case & $\begin{array}{l}\text { Rotational } \\
\text { direction }\end{array}$ & $\begin{array}{l}\text { Rotational } \\
\text { speed (RPM) }\end{array}$ & $\begin{array}{l}\text { Process time } \\
\text { (min) }\end{array}$ & $\begin{array}{l}\text { Percentage of } \\
\text { abrasive (\%) }\end{array}$ & $\begin{array}{l}\text { MRR circular } \\
\left(\mathrm{mm}^{2} / \mathbf{m i n}\right)\end{array}$ & $\begin{array}{l}\text { MRR square } \\
\left(\mathrm{mm}^{2} / \mathrm{min}\right)\end{array}$ & $\begin{array}{l}\text { MRR hexagonal } \\
\left(\mathrm{mm}^{2} / \mathrm{min}\right)\end{array}$ \\
\hline 1 & CW & 100 & 150 & 60 & 0.0126 & 0.0229 & 0.0112 \\
\hline 2 & CW & 100 & 180 & 70 & 0.0099 & 0.0066 & 0.0059 \\
\hline 3 & CW & 100 & 210 & 80 & 0.0060 & 0.0039 & 0.0018 \\
\hline 4 & CW & 125 & 150 & 60 & 0.0059 & 0.0204 & 0.0221 \\
\hline 5 & CW & 125 & 180 & 70 & 0.0036 & 0.0054 & 0.0040 \\
\hline 6 & CW & 125 & 210 & 80 & 0.0030 & 0.0037 & 0.0012 \\
\hline 7 & CW & 150 & 150 & 70 & 0.0127 & 0.0130 & 0.0201 \\
\hline 8 & CW & 150 & 180 & 80 & 0.0070 & 0.0110 & 0.0169 \\
\hline 9 & CW & 150 & 210 & 60 & 0.0179 & 0.0221 & 0.0182 \\
\hline 10 & $\mathrm{CCW}$ & 100 & 150 & 80 & 0.0014 & 0.0033 & 0.0040 \\
\hline 11 & $\mathrm{CCW}$ & 100 & 180 & 60 & 0.0029 & 0.0020 & 0.0059 \\
\hline 12 & $\mathrm{CCW}$ & 100 & 210 & 70 & 0.0018 & 0.0035 & 0.0038 \\
\hline 13 & $\mathrm{CCW}$ & 125 & 150 & 70 & 0.0040 & 0.0039 & 0.0058 \\
\hline 14 & $\mathrm{CCW}$ & 125 & 180 & 80 & 0.0014 & 0.0037 & 0.0046 \\
\hline 15 & $\mathrm{CCW}$ & 125 & 210 & 60 & 0.0193 & 0.0184 & 0.0233 \\
\hline 16 & $\mathrm{CCW}$ & 150 & 150 & 80 & 0.0147 & 0.0188 & 0.0204 \\
\hline 17 & $\mathrm{CCW}$ & 150 & 180 & 60 & 0.0323 & 0.0250 & 0.0285 \\
\hline 18 & CCW & 150 & 210 & 70 & 0.0119 & 0.0152 & 0.0181 \\
\hline
\end{tabular}


Table 8 Internal and external hardness

\begin{tabular}{lllllll}
\hline Test case & $\begin{array}{l}\text { Rotational } \\
\text { direction }\end{array}$ & $\begin{array}{l}\text { Rotational } \\
\text { speed (RPM) }\end{array}$ & $\begin{array}{l}\text { Process } \\
\text { time (min) }\end{array}$ & $\begin{array}{l}\text { Percentage of } \\
\text { abrasive (\%) }\end{array}$ & Internal hardness & $\begin{array}{l}\text { External } \\
\text { hardness }\end{array}$ \\
\hline 1 & CW & 100 & 150 & 60 & 254.16 & 320.14 \\
2 & CW & 100 & 180 & 70 & 257.94 & 314.09 \\
3 & CW & 100 & 210 & 80 & 255.03 & 278.54 \\
4 & CW & 125 & 150 & 60 & 281.06 & 303.63 \\
5 & CW & 125 & 180 & 70 & 268.87 & 322.40 \\
6 & CW & 125 & 210 & 80 & 267.05 & 328.02 \\
7 & CW & 150 & 150 & 70 & 263.64 & 324.07 \\
8 & CW & 150 & 180 & 80 & 265.65 & 312.04 \\
9 & CW & 150 & 210 & 60 & 301.09 & 345.82 \\
10 & CCW & 100 & 150 & 80 & 257.58 & 264.70 \\
11 & CCW & 100 & 180 & 60 & 274.33 & 313.92 \\
12 & CCW & 100 & 210 & 70 & 291.57 & 300.93 \\
13 & CCW & 125 & 150 & 70 & 280.02 & 307.20 \\
14 & CCW & 125 & 180 & 80 & 255.76 & 297.05 \\
15 & CCW & 125 & 210 & 60 & 294.17 & 345.09 \\
16 & CCW & 150 & 150 & 80 & 257.46 & 312.66 \\
17 & CCW & 150 & 180 & 60 & 304.30 & 341.40 \\
18 & CCW & 150 & 210 & 70 & 271.38 & 307.06 \\
\hline
\end{tabular}

Table 7 Surface roughness for internal and external surfaces

\begin{tabular}{|c|c|c|c|c|c|c|c|c|}
\hline Test case & $\begin{array}{l}\text { Rotational } \\
\text { direction }\end{array}$ & $\begin{array}{l}\text { Rotational } \\
\text { speed (RPM) }\end{array}$ & $\begin{array}{l}\text { Process } \\
\text { time (min) }\end{array}$ & $\begin{array}{l}\text { Percentage of } \\
\text { abrasive }(\%)\end{array}$ & $\begin{array}{l}R_{a} \text { internal } \\
\text { circular }(\mu \mathrm{m})\end{array}$ & $\begin{array}{l}R_{a} \text { internal } \\
\text { square }(\mu \mathrm{m})\end{array}$ & $\begin{array}{l}R_{a} \text { internal hex- } \\
\text { agonal }(\mu \mathrm{m})\end{array}$ & $\begin{array}{l}R_{a} \text { external } \\
\text { front }(\mu \mathrm{m})\end{array}$ \\
\hline 1 & CW & 100 & 150 & 60 & 6.819 & 5.818 & 6.672 & 3.446 \\
\hline 2 & CW & 100 & 180 & 70 & 6.513 & 6.825 & 8.102 & 4.405 \\
\hline 3 & CW & 100 & 210 & 80 & 7.331 & 7.333 & 9.596 & 5.119 \\
\hline 4 & CW & 125 & 150 & 60 & 5.574 & 5.839 & 6.695 & 1.882 \\
\hline 5 & CW & 125 & 180 & 70 & 8.052 & 7.029 & 8.156 & 3.355 \\
\hline 6 & CW & 125 & 210 & 80 & 7.803 & 7.458 & 8.660 & 3.841 \\
\hline 7 & CW & 150 & 150 & 70 & 5.255 & 5.997 & 6.681 & 2.931 \\
\hline 8 & CW & 150 & 180 & 80 & 6.171 & 7.706 & 8.246 & 1.918 \\
\hline 9 & CW & 150 & 210 & 60 & 5.424 & 5.804 & 6.069 & 1.228 \\
\hline 10 & $\mathrm{CCW}$ & 100 & 150 & 80 & 7.335 & 9.149 & 8.989 & 6.395 \\
\hline 11 & $\mathrm{CCW}$ & 100 & 180 & 60 & 5.776 & 8.142 & 6.835 & 4.682 \\
\hline 12 & $\mathrm{CCW}$ & 100 & 210 & 70 & 4.867 & 4.318 & 6.075 & 2.354 \\
\hline 13 & CCW & 125 & 150 & 70 & 7.810 & 6.988 & 8.992 & 3.751 \\
\hline 14 & $\mathrm{CCW}$ & 125 & 180 & 80 & 5.437 & 6.774 & 7.624 & 4.039 \\
\hline 15 & $\mathrm{CCW}$ & 125 & 210 & 60 & 6.029 & 6.228 & 6.361 & 2.434 \\
\hline 16 & $\mathrm{CCW}$ & 150 & 150 & 80 & 7.268 & 6.855 & 9.724 & 3.629 \\
\hline 17 & CCW & 150 & 180 & 60 & 4.814 & 5.173 & 5.031 & 1.405 \\
\hline 18 & $\mathrm{CCW}$ & 150 & 210 & 70 & 7.036 & 6.829 & 9.675 & 2.086 \\
\hline
\end{tabular}


Acknowledgements The authors would like to thank Professor Bernard Rolfe for his help in drafting and technical review. All the authors have read and approved the presented materials in the paper and acknowledged the received supports.

Author contribution Dr. Mahyar Khorasani and Dr. Ehsan Farabi contributed to writing and proofreading the manuscript. Mr. AmirHossein Ghasemi helped to draw figures and writing the manuscript. Professor Ian Gibson and Professor Martin Leary contributed to writing. Professor Bernard Rolfe contributed to writing and proofreading the manuscript.

Funding Open Access funding enabled and organized by CAUL and its Member Institutions.

\section{Declarations}

Ethics approval Not applicable.

Consent to participate Not applicable.my

Consent for publication Not applicable.

Conflict of interest The authors declare no competing interests.

Open Access This article is licensed under a Creative Commons Attribution 4.0 International License, which permits use, sharing, adaptation, distribution and reproduction in any medium or format, as long as you give appropriate credit to the original author(s) and the source, provide a link to the Creative Commons licence, and indicate if changes were made. The images or other third party material in this article are included in the article's Creative Commons licence, unless indicated otherwise in a credit line to the material. If material is not included in the article's Creative Commons licence and your intended use is not permitted by statutory regulation or exceeds the permitted use, you will need to obtain permission directly from the copyright holder. To view a copy of this licence, visit http://creativecommons.org/licenses/by/4.0/.

\section{References}

1. Jiang J, Ma Y (2020) Path planning strategies to optimize accuracy, quality, build time and material use in additive manufacturing: a review. Micromachines 11(7):633

2. Jiang J, Xu X, Stringer J (2018) Support structures for additive manufacturing: a review. Journal of Manufacturing and Materials Processing 2(4):64

3. Jiang J et al (2020) Machine learning integrated design for additive manufacturing. J Intell Manuf pp. 1-14

4. Gibson I et al (2020) Additive manufacturing technologies. Third edition, Springer

5. Bourell D et al (2017) Materials for additive manufacturing. CIRP Ann 66(2):659-681

6. Khorasani A et al (2020) A review of technological improvements in laser-based powder bed fusion of metal printers. Int $\mathbf{J}$ Adv Manuf Technol 108(1):191-209

7. Zhao X et al (2021) Effect of process parameters on stress and strain of hybrid deposition and micro-rolling. Rapid Prototyp J

8. Khorasani A et al (2019) The effect of SLM process parameters on density, hardness, tensile strength and surface quality of Ti-6Al4V. Addit Manuf 25:176-186

9. Khorasani AM et al (2019) Investigation on the effect of heat treatment and process parameters on the tensile behaviour of SLM Ti-6Al-4V parts. Int J Adv Manuf Technol 101(9-12):3183-3197
10. Gong $\mathrm{H}$ et al (2014) Analysis of defect generation in Ti-6Al-4V parts made using powder bed fusion additive manufacturing processes. Addit Manuf 1-4:87-98

11. Bisht $\mathrm{M}$ et al (2018) Correlation of selective laser meltingmelt pool events with the tensile properties of Ti-6Al-4V ELI processed by laser powder bed fusion. Addit Manuf 22:302-306

12. Yadroitsev I et al (2018) Qualification of Ti6Al4V ELI alloy produced by laser powder bed fusion for biomedical applications. JOM 70(3):372-377

13. Kumar $\mathrm{M}$ et al (2021) Additive manufacturing of aluminium alloy 2024 by laser powder bed fusion: microstructural evolution, defects and mechanical properties. Rapid Prototyp J

14. de Formanoir $C$ et al (2020) Increasing the productivity of laser powder bed fusion: Influence of the hull-bulk strategy on part quality, microstructure and mechanical performance of Ti-6Al-4V. Addit Manuf 33:p. 101129

15. Khorasani AM et al (2020) Modelling of laser powder bed fusion process and analysing the effective parameters on surface characteristics of Ti-6Al-4V. Int J Mech Sci 168:105299

16. Khorasani A et al (2018) Characterizing the effect of cutting condition, tool path, and heat treatment on cutting forces of selective laser melting spherical component in five-axis milling. J Manuf Sci Eng 140(5)

17. Boschetto A, Bottini L (2015) Surface improvement of fused deposition modeling parts by barrel finishing. Rapid Prototyp J

18. Dixit N, Sharma V, Kumar P (2021) Experimental investigations into abrasive flow machining (AFM) of 3D printed ABS and PLA parts. Rapid Prototyp J

19. Khorasani $\mathrm{M}$ et al (2021) Additive manufacturing a powerful tool for the aerospace industry. Rapid Prototyp J

20. Ferchow $\mathbf{J}$ et al (2020) Model of surface roughness and material removal using abrasive flow machining of selective laser melted channels. Rapid Prototyp J

21. Singh $\mathrm{G}$ et al (2020) Effects of chemically assisted magnetic abrasive finishing process parameters on material removal of Inconel 625 tubes. Procedia Manufacturing 48:466-473

22. Khorasani $\mathrm{M}$ et al (2020) On the role of wet abrasive centrifugal barrel finishing on surface enhancement and material removal rate of LPBF stainless steel 316L. J Manuf Process 59:523-534

23. $\mathrm{Na} \mathrm{W}$ et al (2020) Experiment and simulation analysis on the mechanism of the spindle barrel finishing. Int J Adv Manuf Technol p. $1-18$

24. Lesyk D et al (2020) Post-processing of the Inconel 718 alloy parts fabricated by selective laser melting: Effects of mechanical surface treatments on surface topography, porosity, hardness and residual stress. Surf Coat Technol 381:125136

25. Lesyk D et al (2019) Surface finishing of complexly shaped parts fabricated by selective laser melting. In: Grabchenko's International Conference on Advanced Manufacturing Processes, Springer

26. Nalli F et al (2020) Effect of industrial heat treatment and barrel finishing on the mechanical performance of Ti6Al4V processed by selective laser melting. Appl Sci 10(7):2280

27. Boschetto A et al (2020) Post-processing of complex SLM parts by barrel finishing. Appl Sci 10(4):1382

28. Sood AK, Ohdar R, Mahapatra SS (2009) Improving dimensional accuracy of fused deposition modelling processed part using grey Taguchi method. Mater Des 30(10):4243-4252

29. Rahmati S, Akbari J, Barati E (2007) Dimensional accuracy analysis of wax patterns created by RTV silicone rubber molding using the Taguchi approach. Rapid Prototyp J

30. Gillespie L (2006) Mass finishing handbook. Industrial Press

31. Hou N et al (2021) Fundamental functions of physical and chemical principles in the polishing of titanium alloys: mechanisms and problems. Int J Adv Manuf Technol pp. 1-19 
32. Box G (1988) Signal-to-noise ratios, performance criteria, and transformations. Technometrics 30(1):1-17

33. Warne RT (2014) A primer on multivariate analysis of variance (MANOVA) for behavioral scientists. Pract Assess Res Eval 19

34. Weinfurt KP (1995) Multivariate analysis of variance

35. Henein H, Brimacombe J, Watkinson A (1983) Experimental study of transverse bed motion in rotary kilns. Metall Trans B 14(2):191-205

36. Nalla $\mathrm{R}$ et al (2003) On the influence of mechanical surface treatments-deep rolling and laser shock peening - on the fatigue behavior of Ti-6Al-4V at ambient and elevated temperatures. Mater Sci Eng, A 355(1-2):216-230

37. Wong CC, Hartawan A, Teo WK (2014) Deep cold rolling of features on aero-engine components. Procedia CIRP 13:350-354

38. Zhang $\mathrm{Z}$ et al (2020) Fatigue life enhancement in alpha/beta Ti-6Al-4V after shot peening: An EBSD and TEM crystallographic orientation mapping study of surface layer. Materialia 12:100813

39. Zhang X, Li D, Geng J (2021) An approach to reduce stress and defects: a hybrid process of laser cladding deposition and shot peening. Rapid Prototyp J

40. Lainé SJ et al (2017) Microstructural characterisation of metallic shot peened and laser shock peened Ti-6Al-4V. Acta Mater 123:350-361
41. Sridharan $\mathrm{N}$ et al (2016) Texture evolution during laser direct metal deposition of Ti-6Al-4V. Jom 68(3):772-777

42. Ishfaq K et al (2021) A review on the performance characteristics, applications, challenges and possible solutions in electron beam melted Ti-based orthopaedic and orthodontic implants. Rapid Prototyp J

43. Chicos LA et al (2021) Effect of concentrated solar energy on microstructure evolution of selective laser melted Ti-6Al-4V alloy. Int J Adv Manuf Technol pp. 1-25.

44. Wright SI, Nowell MM, Field DP (2011) A review of strain analysis using electron backscatter diffraction. Microsc Microanal 17(3):316

45. Rotella G et al (2021) Finite element modelling of combined turning/burnishing effects on surface integrity of Ti6Al4V alloy. Int J Adv Manuf Technol pp. 1-11

46. Jiang $X$ et al (2007) Effects of shot-peening and re-shot-peening on four-point bend fatigue behavior of Ti-6Al-4V. Mater Sci Eng, A $468: 137-143$

Publisher's Note Springer Nature remains neutral with regard to jurisdictional claims in published maps and institutional affiliations. 